\title{
TWISTED TORSION ON COMPACT HYPERBOLIC SPACES: A REPRESENTATION-THEORETIC APPROACH*
}

\author{
MARIA G. GARGOVA FUNG ${ }^{\dagger}$
}

\begin{abstract}
In this paper we consider a twisted version $\tau_{\theta}$ of the Ray-Singer analytic torsion on compact locally symmetric spaces $X=K \backslash G / \Gamma$ (with $G$ a noncompact connected semisimple Lie group, $K$ its maximal compact subgroup, and $\Gamma$ a discrete torsion-free cocompact subgroup), where $\theta$ is an automorphism of $X$ with the property that $\theta^{2}=1$. We obtain a representationtheoretic interpretation of the twisted torsion via certain irreducible unitary representations of $G$. By considering $\theta=$ Cartan involution for $S O_{0}(2 n+1,1)$, we show that $\left|\tau_{\theta}\right|=1$ for the compact hyperbolic spaces associated to this family of Lie groups.
\end{abstract}

1. Introduction. The analytic torsion of a compact Riemannian manifold, introduced first by Ray and Singer in [18], is an important invariant that allows us to distinguish between spaces with isomorphic cohomology rings and homotopy groups. It is composed of the spectral information of the Laplacian operators associated to the De Rham complex of the manifold. Cheeger and Müller independently (see [5] and [17]) have shown that this torsion coincides with its combinatorial counterpart, the Reidemeister torsion.

In the special case when the manifold is a compact locally symmetric space $X=$ $K \backslash G / \Gamma$, with $G$ a real semisimple non-compact Lie group, $K$ its maximal compact subgroup and $\Gamma$ a torsion-free cocompact discrete subgroup, Speh in [22] expresses the analytic torsion in terms of representation-theoretic data. Inspired by work of Fried [7] on compact hyperbolic spaces, she describes the spectrum of the Laplacian operators via certain irreducible representations in the unitary dual of $G$. This allows Speh to construct a proof different from that of Moscovici and Stanton [16] of the vanishing of the torsion on all compact locally symmetric spaces of the type above, except in the cases when $G$ has a factor locally isomorphic to $S O(p, q)$ with $p q$ odd, or $S L(3, \mathbb{R})$.

An automorphism of a compact Riemannian manifold acts on the Laplacian operators associated to its complex of differential forms, and thus it acts on the building blocks of the analytic torsion. Therefore, each such automorphism allows us to construct a twisted invariant analogous to the usual torsion. In this paper we do this in the case when $\theta$ is an involution of a compact locally symmetric space. We prove the following vanishing result:

THEOREM 4.2 For the compact locally symmetric space $X=K \backslash G / \Gamma$ with $G$ locally isomorphic to $S O_{0}(2 n+1,1), K$ its maximal compact subgroup and $\Gamma$ a discrete torsion-free subgroup, the twisted torsion

$$
\left|\tau_{\theta}\right|=1
$$

for all outer automorphisms of $G$ such that $\theta^{2}=1$ and $\theta(K)=K, \theta(\Gamma)=\Gamma$.

The proof of this theorem is modeled after Speh's proof of the vanishing of the analytic torsion. Thus, we first obtain a representation-theoretic interpretation of the twisted torsion on a general compact locally symmetric space in 2.3. Then we relate this invariant of the locally symmetric space to "twisted torsion" of $\theta$-invariant unitary

\footnotetext{
${ }^{*}$ Received August 5, 2001; accepted for publication November 16, 2001.

tDepartment of Mathematics, Western Oregon Univeristy, Monmouth, OR 97361, USA. The author would like to extend special gratitude to Birgit Speh for all her direction, encouragement, and patience during the completion of this doctoral research at Cornell University.
} 
representations in the discrete spectrum of $G / \Gamma$. This twisted torsion on representations is distinct from Speh's torsion on representations only when the automorphism $\theta$ is outer.

We specialize to the case when $G$ is locally isomorphic to $S O_{0}(2 n+1,1)$ and $\theta$ is the Cartan involution, since for this Lie group it is essentially (up to inner automorphism) the only outer involution. Moreover, in 3.1 we obtain a reduction of the twisted torsion on unitary representations to that of invariant principal series representations. This allows us to get a formula relating the logarithm of twisted torsion on the manifold to special values of the twisted zeta functions of $\theta$-invariant principal series representations. In sections 4,5 and 6 we show that under the assumptions of the theorem the twisted torsion of all $\theta$-invariant irreducible unitary representations of $G$ is 0 , and hence the logarithm of the twisted torsion on the manifold is 0 .

We work with the disconnected group $\tilde{G} \cong S O_{0}(2 n+1,1) \ltimes\{1, \theta\}$ whose nonidentity component we denote by $G \theta$. We extend each $\theta$-invariant principal series representation $I$ of $G$ by induction to a representation of $\tilde{G}$ with irreducible factor denoted by $\tilde{I}$. We interpret the twisted torsion of such an invariant principal series representation as the coefficient of the trivial representation in a virtual tensor product module (see 4.5), on the $K \theta$ component. At the heart of this character theory computation of the twisted torsion is the calculation of the twisted character $\operatorname{tr} \widetilde{\left(I_{G \theta}\right)}$ performed in section 5 . It turns out that this character is a locally integrable function on the maximal torus of the subgroup $M \cong S O(2 n)$ of $G$.

To get our vanishing result, we complete our proof by recognizing the product of the two characters that define the twisted torsion on representations as products of sums of characters of $S L(2, \mathbb{R})$. To obtain this interpretation, we use combinatorial results in 6 to rewrite, in a suitable form, the characters of those $M$-representations that parametrize the class of $\theta$-invariant principal series for $S O_{0}(2 n+1,1)$.

\section{Twisted Torsion on Compact Locally Symmetric Spaces.}

2.1. Preliminaries. Let $X$ be a compact Riemannian manifold. Denote by $A(X)$ the complex of its $\mathbb{R}$-valued differential forms. By taking the adjoint $d^{*}$ of the differential $D$ we can define the Hodge-Laplacian operator $\Delta_{j}$ on $j$-forms by $\Delta_{j}=D d^{*}+d^{*} D$. This operator is nonnegative and elliptic [24] and we can associate to it the Dirichlet series

$$
\zeta_{\Delta_{j}}=\sum \lambda^{-s}
$$

where we sum over all nonzero eigenvalues of $\Delta_{j}$. This series converges absolutely for $R e(s)$ large enough, and in fact it can be analytically continued to a meromorphic function in the complex plane [21].

We define

$$
\operatorname{det} \Delta_{j}=\exp \left(-\zeta_{\Delta_{j}}^{\prime}(0)\right)
$$

where $\zeta_{\Delta_{j}}^{\prime}$ denotes the first derivative of the zeta function $\zeta_{\Delta_{j}}$.

Definition 2.2. The square of the Ray-Singer analytic torsion [18] $\tau_{1}^{2}$, corresponding to the trivial representation of the fundamental group of $X$, is given by the quotient

$$
\frac{\left(\operatorname{det} \Delta_{1}\right)\left(\operatorname{det} \Delta_{3}\right)^{3} \ldots}{\left(\operatorname{det} \Delta_{2}\right)^{2}\left(\operatorname{det} \Delta_{4}\right)^{4} \ldots}
$$


We consider only compact locally symmetric spaces of the following kind: $X=$ $K \backslash G / \Gamma$, where $G$ is a semisimple noncompact connected real Lie group with maximal compact subgroup $K$ and $\Gamma$ is a discrete torsion-free cocompact subgroup of $G$.

The Lie algebra $\mathfrak{g}$ of $G$ has the Cartan decomposition $\mathfrak{g}=\mathfrak{k} \oplus \mathrm{p}$, where $\mathfrak{k}$ is the Lie algebra of $K$ and $\mathrm{p} \cong T(X)_{e}$, the tangent space at the identity of $X$.

For every $(\mathfrak{g}, \mathfrak{k})$-module $M$ of the universal enveloping algebra $U(\mathfrak{g})$ of $\mathfrak{g}$ we can consider the relative Lie algebra complex

$$
\mathbf{C}^{*}(\mathfrak{g}, M)=\operatorname{Hom}_{\mathfrak{k}}\left(\wedge^{*} \mathrm{p}, M\right)
$$

where we consider $\mathrm{p}$ as a $\mathfrak{k}$-module via the adjoint action. If $M$ is unitary, there is a natural inner product on $\operatorname{Hom}_{\mathfrak{e}}\left(\wedge^{*} p, M\right)$ coming from the Killing form on $\mathrm{p}$ and the inner product on $M$. Thus, following the exposition of Chapter 2 in [2], we can define a Laplace operator

$$
\Delta_{M}^{j}: \operatorname{Hom}_{\mathfrak{k}}\left(\wedge^{j} \mathrm{p}, M\right) \rightarrow \operatorname{Hom}_{\mathfrak{k}}\left(\wedge^{j} \mathrm{p}, M\right)
$$

Kuga's lemma (page 49 in [2]) then gives us that for each $\omega \in \wedge^{j} \mathrm{p}^{*}$ and $v \in M$ (recall that $\mathbf{C}^{j}(\mathfrak{g}, M) \cong\left[\left(\wedge^{j} \mathrm{p}\right)^{*} \otimes M\right]^{\mathfrak{k}}$, where we take the $\mathfrak{k}$-invariants in the latter term)

$$
\Delta_{M}^{j}(\omega \otimes v)=\omega \otimes(-C v)
$$

with $C$ being the Casimir operator associated to the module $M$. On the other hand, $A(X)$ is isomorphic to $\operatorname{Hom}_{\mathfrak{k}}\left(\wedge^{*} \mathrm{p}, C^{\infty}(G / \Gamma)\right)$, with $C^{\infty}(G / \Gamma)$ a module for the universal enveloping algebra $U(\mathfrak{g})$ via right invariant differentiation. Thus the spectrum of the Laplace operator is in one-to-one correspondence with the spectrum of the Casimir operator $C$ on $L^{2}(G / \Gamma)$.

Fix $d x$ to be the Haar measure on $G / \Gamma$ coming from the Haar measure on $G$. Then the Hilbert space $L^{2}(G / \Gamma)$ of square integrable functions on $G / \Gamma$ with respect to $d x$ is the completion of the space $C^{\infty}(G / \Gamma)$. Moreover, we can decompose $L^{2}(G / \Gamma)$ as a discrete sum of irreducible $G$-modules with finite multiplicities, by a theorem of Gel'fand and Piateckii-Shapiro [9]:

$$
\dot{L}^{2}(G / \Gamma) \cong \oplus m(\pi, \Gamma) H_{\pi}
$$

where we sum over all irreducible unitary representations $\left(\pi, H_{\pi}\right)$ in the unitary dual $\hat{G}_{u}$ and $m(\pi, \Gamma)=\operatorname{dim} \operatorname{Hom}_{G}\left(H_{\pi}, L^{2}(G / \Gamma)\right)$.

Hence it follows easily (see [22]) that for $\lambda \neq 0 \in \mathbb{R}$,

$$
\operatorname{dim} \operatorname{ker}\left(\Delta_{j}-\lambda\right)=\sum_{\substack{\pi \in \hat{G}_{u} \\ \pi(C)=\lambda}} m(\pi, \Gamma) \operatorname{dim} \operatorname{Hom}_{\mathfrak{k}}\left(\wedge^{j} \mathrm{p}, H_{\pi}^{\infty}\right)
$$

where $H_{\pi}^{\infty}$ denotes the $C^{\infty}$-vectors in $H_{\pi}$. We denote the dimension of this kernel by $m(\lambda, j, \Gamma)$.

This allows us to write

$$
\log \tau_{1}^{2}=\lim _{s \rightarrow 0} \sum_{j}(-1)^{j} j \zeta_{\Delta_{j}}^{\prime}(s) \quad \text { where } \quad \zeta_{\Delta_{j}}=\sum m(\lambda, j, \Gamma) \lambda^{-s} .
$$


2.3. Definition of the Twisted Torsion and Representation-Theoretic Interpretation. Let us fix an arbitrary automorphism $\theta$ of the Lie group $G$ such that $\theta(K)=K, \theta(\Gamma)=\Gamma$ and $\theta^{2}=1$. We can think of $\theta$ as an automorphism of $X=K \backslash G / \Gamma$, by identifying it with the induced map on the locally symmetric space.

The automorphism $\theta$ acts on the complex of differential forms $A(X)$, which we identify with the space $\operatorname{Hom}_{\mathfrak{k}}\left(\wedge^{*} \mathrm{p}, C^{\infty}(G / \Gamma)\right)$, in the following way:

$$
\theta \cdot \eta\left(Y_{1}, Y_{2}, \ldots, Y_{q}\right)(x)=\eta\left(d \theta Y_{1}, d \theta Y_{2}, \ldots, d \theta Y_{q}\right)(\theta(x))
$$

with $\eta \in \operatorname{Hom}_{\mathfrak{k}}\left(\wedge^{q} \mathrm{p}, C^{\infty}(G / \Gamma)\right), Y_{1}, \ldots, Y_{q} \in \mathrm{p}$ and $x \in G / \Gamma$. Another way of writing this $\mathfrak{k}$-covariant action is:

$$
\theta \cdot \eta=\eta^{\theta}=\theta_{C^{\infty}(G / \Gamma)}^{-1} \circ \eta \circ \theta_{\wedge q}
$$

with $\theta_{C^{\infty}(G / \Gamma)}$ and $\theta_{\wedge^{q} \mathrm{p}}$ denoting the action of $\theta$ on $C^{\infty}(G / \Gamma)$ and $\wedge^{q} \mathrm{p}$, respectively.

The automorphism $\theta$ acts on the Laplacian operator $\Delta_{q}$ by

$$
\Delta_{q}^{\theta}=\theta \cdot \Delta_{q}(\eta)=\theta\left(\Delta_{q}\left(\theta^{-1} \cdot \eta\right)\right)=\theta\left(\Delta_{q}(\theta \cdot \eta)\right)
$$

since the Laplacian is a linear map.

DEFINITION 2.4. The square of the twisted torsion $\tau_{\theta}^{2}$ corresponding to the trivial representation of $\Gamma$ is

$$
\frac{\left(\operatorname{det} \Delta_{1}^{\theta}\right)\left(\operatorname{det} \Delta_{3}^{\theta}\right)^{3} \ldots}{\left(\operatorname{det} \Delta_{2}^{\theta}\right)^{2}\left(\operatorname{det} \Delta_{4}^{\theta}\right)^{4} \ldots}
$$

We would like to obtain a representation-theoretic interpretation of the twisted torsion on the compact locally symmetric space $X$. As a first step in this direction we get the analogue of Kuga's lemma.

We call a unitary $(\mathfrak{g}, \mathfrak{k})$-module $(\pi, M) \theta$-invariant if the module $\left(\pi^{\theta}, M\right)$ is isomorphic to the original module, with $\pi^{\theta}(X) \cdot m=\pi(d \theta(X)) \cdot m$ for $X \in \mathfrak{g}, m \in M$.

LEMMA 2.5. Let $(\pi, M)$ be a unitary, $\theta$-invariant $(\mathfrak{g}, \mathfrak{k})$-module with corresponding Laplacian operator $\Delta(\pi)$. If $\eta \in C^{q}(M)=\operatorname{Hom}_{\mathfrak{k}}\left(\wedge^{q} \mathrm{p}, M\right)$, then

$$
\left(\Delta(\pi) \eta^{\theta}\right)_{I}=-\pi(C) \eta_{I}^{\theta}
$$

where $C$ denotes the Casimir element associated to $(\pi, M)$ and $\eta^{\theta}=\theta_{M}^{-1} \circ \eta \circ \theta_{\wedge q \mathrm{p}}$ by $\eta^{\theta}$.

Proof. The same proof as the one on p.49 in [2] goes through, with $\eta$ replaced

Corollary 2.6. $\Delta(\pi)^{\theta}(\omega \otimes v)=\omega \otimes \theta(-C(\theta \cdot v))$ for $\omega \in \wedge^{*} \mathrm{p}^{*}$ and $v \in M$.

Proof. By the above lemma we have that

$$
\theta\left(\Delta(\pi)\left(\omega^{\theta} \otimes v^{\theta}\right)\right)=\theta\left(\omega^{\theta} \otimes-C(\theta \cdot v)\right)=\omega \otimes \theta(-C(\theta \cdot v))
$$

If $\theta\left(-C\left(v^{\theta}\right)\right)=\lambda v$ for some $\lambda \neq 0$, then $-C\left(v^{\theta}\right)=\lambda v^{\theta}$. Conversely, if $C\left(v^{\theta}\right)=$ $\lambda v^{\theta}$, then $\theta\left(-C\left(v^{\theta}\right)\right)=-\lambda v$. Thus, the spectrum of the twisted Laplacian depends on the spectrum of the Casimir element $C$. 
Recall now the decomposition of the unitary $G$-module $L^{2}(G / \Gamma)$ whose $C^{\infty}$ vectors are $C^{\infty}(G / \Gamma)$ (see [9])

$$
L^{2}(G / \Gamma) \cong \oplus m(\pi, \Gamma) H_{\pi},
$$

where we sum over all irreducible unitary representations $\left(\pi, H_{\pi}\right)$ in the unitary dual $\hat{G}_{u}$ and $m(\pi, \Gamma)=\operatorname{dim}_{H_{0}}\left(H_{\pi}, L^{2}(G / \Gamma)\right)$. The map $\theta$ acts on the left side of equation (2.3) by

$$
\theta \cdot f(x)=f(\theta(x))
$$

for all $f \in L^{2}(G / \Gamma)$ and $x \in G / \Gamma$. Obviously, $\theta$ leaves $L^{2}(G / \Gamma)$ invariant.

Consider now the right side of equation (2.3). There are two possibilities that can occur. First, it can happen that the representation $H_{\pi}$ is sent by the action of $\theta$ to a representation isomorphic to it. In this case, there exists an intertwining operator $A_{\theta}: H_{\pi} \mapsto H_{\pi}$ such that

$$
\begin{gathered}
A_{\theta} \pi(g)=\pi(\theta(g)) A_{\theta} \\
A_{\theta}^{2}=1 .
\end{gathered}
$$

Notice that the operator $A_{\theta}$ is thus determined up to a sign, $\epsilon_{\theta}$. In this situation, $\theta$ also acts on $\alpha \in \operatorname{Hom}_{G}\left(H_{\pi}, L^{2}(G / \Gamma)\right)$ by

$$
\theta \cdot \alpha=\theta_{L^{2}(G / \Gamma)}^{-1} \circ \alpha \circ \theta_{H_{\pi}}
$$

with $\theta_{L^{2}(G / \Gamma)}$ and $\theta_{H_{\pi}}$ denoting the actions of $\theta$ on $L^{2}(G / \Gamma)$ and $H_{\pi}$ respectively. Note that this action is $G$-covariant.

Second, it can happen that $\theta$ sends the representation $H_{\pi}$ into a representation $H_{\pi}^{\theta}$ which is not isomorphic to it. In this case, we can look at the direct sum $H_{\pi} \oplus H_{\pi}^{\theta}$. Clearly, this sum is invariant under $\theta$. Moreover, we can show the following.

LEмма 2.7. If $H_{\pi} ¥ H_{\pi}^{\theta}$, then the trace of $\theta$,

$$
\left.\operatorname{tr} \theta\right|_{\operatorname{Hom}_{\mathfrak{e}}\left(\wedge^{j} \mathrm{p}, H_{\pi}^{\infty} \oplus\left(H_{\pi}^{\infty}\right)^{\theta}\right)}=0 \text { for all } j \text {. }
$$

Proof. We can choose a basis of the finite dimensional vector space $\operatorname{Hom}_{\mathfrak{k}}\left(\wedge^{j} \mathrm{p}, H_{\pi}^{\infty} \oplus\left(H_{\pi}^{\infty}\right)^{\theta}\right)$ consisting of $\mathfrak{k}$-homomorphisms $\alpha_{1}, \ldots, \alpha_{l}$ which live on $\left(H_{\pi}^{\infty}\right)^{\theta}$ and are trivial on $H_{\pi}^{\infty}$, for some positive integer $l$, and $\mathfrak{k}$-homomorphisms $\beta_{1}, \ldots, \beta_{m}$ which live on $H_{\pi}^{\infty}$ and are trivial on $\left(H_{\pi}^{\infty}\right)^{\theta}$, for some positive integer $m$. Since $\theta$ is an invertible linear map with the property $\theta^{2}=1$, it follows that in fact $l=m$. Moreover, $\theta$ maps each $\alpha_{i}$ into $\sum_{j} c_{j} \beta_{j}(1 \leq j \leq l)$ for some nonzero constants $c_{j}$, and hence its trace on $\operatorname{Hom}_{\mathfrak{k}}\left(\wedge^{j} \mathrm{p}, H_{\pi}^{\infty} \oplus\left(H_{\pi}^{\infty}\right)^{\theta}\right)$ is 0 .

Theorem 2.8. Suppose that $G / \Gamma$ is compact and let $C$ be the Casimir element in the universal enveloping algebra $U(\mathfrak{g})$. For $\lambda \neq 0 \in \mathbb{R}$

$$
\operatorname{dim} \operatorname{ker}\left(\Delta_{j}^{\theta}-\lambda\right)=\left.\left.\sum_{\substack{\pi \in \hat{G}_{u} \\ \pi(C)=\lambda}} \operatorname{tr} \theta\right|_{\operatorname{Hom}_{G}\left(H_{\pi}, L^{2}(G / \Gamma)\right)} \operatorname{tr} \theta\right|_{\operatorname{Hom}_{\mathfrak{e}}\left(\wedge^{j} \mathrm{p}, H_{\pi}^{\infty}\right)} .
$$

Proof. Since the operator $\Delta_{j}$ is elliptic, so is its twisted version $\Delta_{j}^{\theta}$. This implies that we can write $A^{j}(X)$ as a direct sum of its eigenspaces. By the corollary to 
Lemma 2.5, the action of the twisted Laplace operator corresponds to the action of the Casimir element on $C^{\infty}(G / \Gamma)$. By the above analysis, it follows that the decomposition claimed in the theorem is precisely the eigenspace decomposition.

REMARK. This theorem implies that if we want to calculate the twisted torsion on $X$ it suffices for us to consider only the unitary irreducible representations $\left(\pi, H_{\pi}\right)$ and their corresponding modules $H_{\pi}^{\infty}$ of $C^{\infty}$-vectors which are $\theta$-invariant.

Corollary 2.9. Suppose that $X=K \backslash G / \Gamma$ is a compact locally symmetric space and let $\operatorname{tr}(\lambda, j, \Gamma)=\left.\left.\sum_{\substack{\pi \in \hat{G}_{u} \\ \pi(C)=\lambda}} \operatorname{tr} \theta\right|_{\operatorname{Hom}_{G}\left(H_{\pi}, L^{2}(G / \Gamma)\right)} \operatorname{tr} \theta\right|_{\operatorname{Hom}_{\mathfrak{e}}\left(\wedge^{j} \pi, H_{\pi}^{\infty}\right)}$. Then

$$
\zeta_{\Delta_{j}^{\theta}}(s)=\sum_{\lambda} \operatorname{tr}(\lambda, j, \Gamma) \lambda^{-s} \quad \text { for } \quad s \in \mathbb{C}
$$

Furthermore,

$$
\log \tau_{\theta}^{2}=\lim _{s \rightarrow 0} \sum_{j}(-1)^{j} j{\zeta_{\Delta_{j}^{\theta}}^{\prime}}^{\prime}(s)
$$

Proof. Since $\left|\zeta_{\Delta_{j}^{\theta}}\right|$ is dominated by $\left|\zeta_{\Delta_{j}}\right|$ for the zeta function of the usual torsion, it converges for $\operatorname{Re}(s)$ large enough (the classical case is shown in [10]) and can be extended to a meromorphic function on $\mathbb{C}$. Hence, the two equations in the corollary make sense (see also equation (2.1)). The fact that they hold is obvious.

In view of this result, it is reasonable to consider the following functions on $(\mathfrak{g}, \mathfrak{k})$ modules.

Definition 2.10. Let $(\rho, M)$ be a $\theta$-invariant $(\mathfrak{g}, \mathfrak{k})$-module. Define the torsion $\operatorname{tor}(\rho)$ and its twisted version $\operatorname{tor}^{\theta}(\rho)$ to be

$$
\begin{gathered}
\operatorname{tor}(\rho)=\sum_{j}(-1)^{j} j \operatorname{dim} \operatorname{Hom}_{\mathfrak{k}}\left(\wedge^{j} \mathrm{p}, M\right) \\
\operatorname{tor}^{\theta}(\rho)=\left.\sum_{j}(-1)^{j} j \operatorname{tr} \theta\right|_{\operatorname{Hom}_{\mathfrak{e}}\left(\wedge^{j} \mathrm{p}, M\right)} .
\end{gathered}
$$

We would like to find conditions under which these two functions coincide.

Proposition 2.11. Suppose that $\theta$ is an automorphism of $X$ which comes from an inner automorphism on $G$, and let $(\rho, M)$ be a $\theta$-invariant $(\mathfrak{g}, \mathfrak{k})$-module. Then

$$
|\operatorname{tor}(\rho)|=\left|\operatorname{tor}^{\theta}(\rho)\right| \text {. }
$$

Proof. The automorphism $\theta(g)=\alpha g \alpha^{-1}$ is defined by an element $\alpha \in G$ of finite order. This means that $\alpha \in K$. Denote by $X_{\alpha}$ the corresponding element in $\mathfrak{k}$. Let $\omega \in \operatorname{Hom}_{\mathfrak{k}}\left(\wedge^{j} \mathrm{p}, M\right)$ and $Y \in \wedge^{j} \mathrm{p}$. Then

$$
\theta \cdot(\omega(Y))=A_{\theta}^{-1} \omega^{\theta}(Y)=A_{\theta}^{-1} \omega\left(\operatorname{ad}\left(X_{\alpha}\right) Y\right)=A_{\theta}^{-1} \rho\left(X_{\alpha}\right) \omega(Y) .
$$

Now we can choose $A_{\theta}=\rho\left(X_{\alpha}\right)$.

Corollary 2.12. Assume $\theta$ is an automorphism of $X$ coming from an automorphism of the group $G$ of the form $\theta=\theta_{1} \circ \theta_{2}$ where $\theta_{1}$ is an outer automorphism and $\theta_{2}$ is an inner automorphism. Then for $\theta$-invariant $(\mathfrak{g}, \mathfrak{k})$-modules $(\rho, M)$,

$$
\left|\operatorname{tor}^{\theta}(\rho)\right|=\left|\operatorname{tor}^{\theta_{1}}(\rho)\right| \text {. }
$$

Proof. This is immediate from proposition 2.11 and the multiplicative property of the trace. 
3. Twisted Torsion on Representations of $S O_{0}(2 n+1,1)$. From this point on we are going to assume that the Lie group $G$ is isomorphic to the connected group $S O_{0}(2 n+1,1)$.

3.1. Reduction to the Principal Series Case. Let $P=M A N$ be a minimal parabolic subgroup of $G, \sigma$ an irreducible representation of $M \cong S O(2 n)$, and $\nu$ a character of $\operatorname{Lie}(A)=\mathfrak{a}$. Denote the principal series representation $\operatorname{Ind}_{P}^{G}(\sigma \otimes \nu \otimes 1)$ by $(I(P, \sigma, \nu), \pi)$. The action of $G$ here is by $\pi(x) f(g)=f\left(x^{-1} g\right)$ for $x, g \in G$, $f \in I=I(P, \sigma, \nu)$.

For an admissible representation $U$ such that $U^{\theta}=U \circ \theta \cong U$ and an automorphism $\theta$ of $G$ with the property that $\theta^{2}=1$, recall the following definition (in view of 2.10):

DeFInITION 3.2. The twisted torsion of $U$ with respect to $\theta$ is

$$
\operatorname{tor}^{\theta}(U)=\left.\sum_{i}(-1)^{i} i \operatorname{tr} \theta\right|_{\operatorname{Hom}_{K}\left(\wedge^{i} \mathrm{p}, U\right)}
$$

We observe that the twisted torsion $\operatorname{tor}^{\theta}(I(P, \sigma, \nu))$ is independent of the character $\nu$ since it depends only on the $K$-type structure of $I(P, \sigma, \nu)$. Moreover, as in lemma 2.7 , if $U^{\theta}$ is not isomorphic to $U$, we can show that

$$
\left.\operatorname{tr} \theta\right|_{\operatorname{Hom}_{K}\left(\wedge^{i} \mathrm{p}, U \oplus U^{\theta}\right)}=0 .
$$

Now we can explore the vanishing properties of $\tau_{\theta}$ on the associated locally symmetric space, by considering only the twisted torsion of $\theta$-invariant modules of $G$.

LEMma 3.3. Let $U$ be an admissible, $\theta$-invariant representation of $G$. Then the twisted torsion $\operatorname{tor}^{\theta}(U)$ is completely determined by $\operatorname{tor}^{\theta}(I(P, \sigma, \nu))$ for the principal series representations which appear in the unique decomposition of $U$ in the Grothendieck group of $G$.

Proof. Write $U$ in the Grothendieck group uniquely as

$$
U=\sum m(U, \sigma \otimes \nu) I(P, \sigma, \nu)
$$

where $m(U, \sigma \otimes \nu)$ are integral coefficients. As before, we may assume that $I \cong I^{\theta}$, because $U$ is $\theta$-invariant. Then the action of $\theta$ on $U$ is completely determined by the operator $A_{\theta}$ acting on $I$. The fact that the twisted torsion of a principal series is independent of the parameter $\nu$, together with the linearity of the trace, then gives us the required result.

Proposition 3.4. Suppose that $X$ is the compact locally symmetric space associated to $G$. Then

$$
\sum_{j}(-1)^{j} j \zeta_{\Delta_{j}}^{\theta}(s)=\left.\sum_{U \in \hat{G}_{u}^{\prime}} \operatorname{tor}^{\theta}(U) \operatorname{tr} \theta\right|_{m(U, \Gamma)} U(C)^{-s}
$$

where $\widehat{G_{u}^{\prime}}$ denotes the collection of all $\theta$-invariant irreducible unitary representations on which the Casimir element $C$ has a nonzero eigenvalue.

Proof. This follows from corollary 2.9 since the series are absolutely convergent for $R e(s)$ large enough.

Now we can conclude that 
COROLlaRY 3.5. If the twisted torsion $\operatorname{tor}^{\theta}(I)$ is zero for all $\theta$-invariant principal series representations of $G$, then the twisted torsion $\tau_{\theta}^{2}(X)$ equals 1 on the associated locally symmetric space $X=K \backslash G / \Gamma$.

Proof. By (3.3) and (3.4) it follows that the logarithm of $\tau_{\theta}^{2}(X)$ is 0 .

3.6. The Twisted Principal Series of $S O_{0}(2 n+1,1)$. The only outer automorphism (up to composition with an inner one) for $S O_{0}(2 n+1,1)$ is the Cartan involution (see [19]), and thus from now on we will fix $\theta$ to be exactly this map. It is explicitly given by conjugation with the $(2 n+2) \times(2 n+2)$ matrix

$$
\left(\begin{array}{ccccc}
-1 & 0 & \ldots & 0 & 0 \\
0 & -1 & \ldots & 0 & 0 \\
\vdots & \vdots & \ddots & \vdots & \vdots \\
0 & 0 & \ldots & -1 & 0 \\
0 & 0 & \ldots & 0 & 1
\end{array}\right)
$$

with $2 n+1(-1)$ 's down the diagonal. We denote by $\theta$ the Cartan involution, both on the group and Lie algebra level.

We want to calculate $\operatorname{tor}^{\theta}(I(P, \sigma, \nu))=\operatorname{tor}^{\theta}(I)$. Recall that $\theta$ acts on $\alpha \in$ $\operatorname{Hom}_{K}\left(\wedge^{i} \mathrm{p}, I\right)$ by

$$
\alpha^{\theta}=\theta_{I}^{-1} \circ \alpha \circ \theta_{\wedge^{i} \mathrm{p}}
$$

where $\theta_{I}$ and $\theta_{\wedge^{i} \mathrm{p}}$ denote the actions of $\theta$ on $I$ and $\wedge^{i} \mathrm{p}$, respectively. The Cartan involution acts by -1 on the whole subspace $p$, and thus its action on $\wedge^{i} \mathrm{p}$ is given by $(-1)^{i}$. The action of $\theta$ on $(I, \pi)$ is determined by an intertwining operator $A_{\theta}$, known up to a sign, with the properties that

$$
A_{\theta} \pi(x)=\pi(\theta(x)) A_{\theta} \text { and } A_{\theta}^{2}=1
$$

for all $x \in G$.

We want to obtain an explicit formula for $A_{\theta}$. As noted above, we assume that $I^{\theta} \cong I$. First we analyze the twisted representation $I^{\theta}=I \circ \theta$. A calculation shows that we can identify $I^{\theta}$ with the principal series $I(\bar{P}, \sigma,-\nu)$, where $\bar{P}=\theta(P)$. We would like this principal series to be isomorphic to $I$. By the theory of intertwining operators (see [13], [14]) this will happen if there exists an element $w_{0} \in N_{K}(A)$ (in fact, $w_{0}$ here is a representative for an element of the Weyl group $W(G, A)$ ) such that $w_{0} \circ \sigma \cong \sigma, w_{0} \circ \theta \nu=\nu$ and $w_{0}^{-1}(\bar{P})=P$. Furthermore, we can explicitly exhibit the map Int: $I^{\theta} \rightarrow I$ :

$$
f(g) \mapsto \psi(g)=a_{\theta} f\left(g w_{0}^{-1}\right)
$$

for all $g \in G$. Here $a_{\theta}$ is defined to be a map (again determined up to a sign) $a_{\theta}: V_{w_{0} \theta \sigma} \rightarrow V_{\sigma}$ between the $M$-modules with the same underlying vector space and different actions, encoded by the corresponding subscripts (i.e. for $v \in V_{w_{0} \theta \sigma}$, $\left.m \cdot v=\sigma\left(w_{0} \theta(m)\right) \cdot v\right)$. This operator has the following properties:

$$
a_{\theta} \sigma(m)=\sigma\left(w_{0} \theta(m)\right) a_{\theta} \text { and } a_{\theta}^{2}=1 .
$$

Now, we can compose the two maps Int $\circ \theta$ to obtain a map $A_{\theta}: I \rightarrow I$. 
LEMMA 3.7. The operator $A_{\theta}: I \rightarrow I$ such that for $f \in I$,

$$
A_{\theta} f(g)=a_{\theta} f\left(\theta(g) w_{0}^{-1}\right)
$$

gives explicitly the action of $\theta$ on the principal series $I=(I(P, \sigma, \nu), \pi)$.

Proof. It is trivial to check that $A_{\theta}^{2}=1$ and $A_{\theta} \pi(g)=\pi(\theta(g)) A_{\theta}$. Consider $\hat{f}(x \operatorname{man})=A_{\theta} f(x \operatorname{man})$, for $x \in G, m \in M, a \in A$ and $n \in N$.

$$
\begin{aligned}
\hat{f}(x \operatorname{man}) & =a_{\theta} f\left(\theta(x \operatorname{man}) w_{0}^{-1}\right) \\
& =a_{\theta} f\left(\theta(x) w_{0}^{-1} m^{w_{0}} a^{-w_{0}} n\right) \\
& =a_{\theta} e^{-\left(\nu+\rho_{I}\right) \log a^{-w_{0}}} \sigma\left(m^{w_{0}}\right)^{-1} f\left(\theta(x) w_{0}^{-1}\right) \\
& =e^{w_{0}\left(\nu+\rho_{I}\right) \log a} a_{\theta} \sigma\left(w_{0} \circ \theta(m)\right)^{-1} f\left(\theta(x) w_{0}^{-1}\right) \\
& =e^{-\left(\nu+\rho_{I}\right) \log a} \sigma(m)^{-1} a_{\theta} f\left(\theta(x) w_{0}^{-1}\right)
\end{aligned}
$$

with $g^{w_{0}}$ denoting $w_{0} g w_{0}^{-1}$ and by $w_{0} \circ \theta(n)=n$ since $f \in I$

by the definition of $a_{\theta}$ and $w_{0} \circ \theta \nu=\nu$.

Thus $\hat{f}$ belongs to $I$.

REMARK. If we assume the existence of $w_{0}$, then the class of $\theta$-invariant principal series is determined by the class of tempered representations on $M$ with the property that $w_{0} \circ \sigma \cong \sigma$. In the case that we are interested in, this translates into the class of irreducible representations of $M$ such that $w_{0} \circ \sigma \cong \sigma$.

For $G \cong S O_{0}(2 n+1,1)$, the element $w_{0}$ can be chosen to be the $(2 n+2) \times(2 n+2)$ matrix

$$
\left(\begin{array}{cccccc}
-1 & 0 & 0 & 0 & \ldots & 0 \\
0 & 0 & 1 & 0 & \ldots & 0 \\
0 & 1 & 0 & 0 & \ldots & 0 \\
0 & 0 & 0 & 1 & \ldots & 0 \\
\vdots & \vdots & \vdots & \vdots & \ddots & \vdots \\
0 & 0 & 0 & 0 & \ldots & 1
\end{array}\right)
$$

with $(2 n-1)$ 1's down the diagonal, starting at the 4 th row. This choice is based on selecting, for the maximal abelian subspace $\mathfrak{a}$ of $\mathrm{p}$, the set of $(2 n+2) \times(2 n+2)$ matrices $\left(b_{i, j}\right)$ whose only nonzero entries are the $b_{1,2 n+2}=b_{2 n+2,1}$. By Frobenius reciprocity we have that for all $i$

$$
\operatorname{Hom}_{K}\left(\wedge^{i} \mathrm{p}, I(P, \sigma, \nu)\right) \cong \operatorname{Hom}_{M}\left(\wedge^{i} \mathrm{p}, V\right),
$$

with $(\sigma, V)$ denoting an irreducible representation of $M$. Thus we have to consider only those representations of $M$ that appear in the exterior powers of the $(2 n+1)$ dimensional vector space $\mathrm{p}$. This is the set of $M$-modules consisting of the trivial representation $T_{t r}$, the standard representation $V_{s t}$ of degree $2 n, \wedge^{i} V_{s t}$ for $2 \leq i \leq$ $(n-1)$, and the two irreducible representations $V_{1}, V_{2}$ of degree $\frac{1}{2}\left(\begin{array}{c}2 n \\ n\end{array}\right)$ into which $\wedge{ }^{n} V_{s t}$ splits. As pointed out on p.185 in [2], the action of $w_{0}$ sends $V_{1}$ exactly into $V_{2}$, and since we know these two modules are not isomorphic to each other, we should not take them into account when determining the value of the twisted torsion. 
Therefore, in calculating the twisted torsion for the principal series $I=I(P, \sigma, \nu)$ for $S O_{0}(2 n+1,1)$, the set of $\theta$-invariant modules $I$ is exhausted by those with $\sigma$ parameters in the set of $w_{0}$-invariant irreducible $M$-modules given by:

$$
\left\{T_{t r}, V_{s t}, \wedge^{i} V_{s t} \mid \text { for } 2 \leq i \leq(n-1)\right\} \text {. }
$$

3.8. Example. Let $G=S O_{0}(3,1)$. Then we have that $K \cong S O(3)$ and $M \cong$ $S O(2)$. By (3.2) we only need consider the trivial $M$-module $\left(T_{t r}, t r i v\right)$. Hence, $\operatorname{tor}^{\theta}(I(P, \operatorname{triv}, \nu))=\operatorname{tor}^{\theta}(I)$ equals

$$
-\left.\operatorname{tr} \theta\right|_{\operatorname{Hom}_{K}(\mathrm{p}, I)}+\left.2 \operatorname{tr} \theta\right|_{\operatorname{Hom}_{K}\left(\wedge^{2} \mathrm{p}, I\right)}-\left.3 \operatorname{tr} \theta\right|_{\operatorname{Hom}_{K}\left(\wedge^{3} \mathrm{p}, I\right)} .
$$

Since the trivial representation of $M$ appears exactly once in each of the exterior powers of $\mathrm{p}$ when considered as $M$-modules, it follows that

$\operatorname{dim} \operatorname{Hom}_{M}\left(\wedge^{i} \mathrm{p}, T_{t r}\right)=1$ for all $i$. Let $E_{j}$ be the basis element of $\mathrm{p}$ whose only nonzero $(4, j)$-th and $(j, 4)$-th entries are 1 , for $j=1,2,3$. Then a basis of $\operatorname{Hom}_{K}\left(\mathrm{p}, T_{t r}\right)$ is given by $\alpha_{M}$ such that $\alpha_{M}\left(E_{1}\right)=v \neq 0 \in T$ and $\alpha_{M}\left(E_{2}\right)=\alpha_{M}\left(E_{3}\right)=0$. We can pull back $\alpha_{M}$ to a homomorphism $A \in \operatorname{Hom}_{K}(\mathrm{p}, I)$ such that for $X \in \mathrm{p}$ and $k \in K$,

$$
A(X)(k)=\alpha_{M}\left(\rho(k)^{-1} X\right),
$$

where $\rho$ denotes the representation of $K$ on $\mathrm{p}$ given by conjugation. By choosing $a_{\theta}=1$, the action of $\theta$ maps $A(X)$ into

$$
\begin{aligned}
\theta_{I} \circ A(X) \circ \theta_{\mathrm{p}}=A_{\theta}(A(-X))(k) & =-A(X)\left(k\left(w_{0}\right)^{-1}\right) \\
& =-\alpha_{M}\left(\rho\left(k w_{0}\right)^{-1} X\right)=-\alpha_{M}\left(\left(k w_{0}\right)^{t} X\right) .
\end{aligned}
$$

The multiplication $\left(k w_{o}\right)^{t}$ negates the entries in the first row of the element $k^{t}$ and thus produces a negation of $X$. Hence, we get that

$$
\left.\operatorname{tr} \theta\right|_{\operatorname{Hom}_{K}(\mathrm{p}, I)}=(-1)(-1)=1 .
$$

A completely analogous calculation shows that

$$
\begin{aligned}
& \left.\operatorname{tr} \theta\right|_{\operatorname{Hom}_{K}\left(\wedge^{2} \mathrm{p}, I\right)}=-1 \\
& \left.\operatorname{tr} \theta\right|_{\mathrm{Hom}_{K}\left(\wedge^{3} \mathrm{p}, I\right)}=-1
\end{aligned}
$$

and thus we get that

$$
\operatorname{tor}^{\theta}(I)=-1+2(-1)-3(-1)=0 .
$$

Therefore, we have shown that the twisted torsion vanishes for all principal series representations of $S O_{0}(3,1)$ and thus $\left|\tau_{\theta}\right|=1$ on the associated locally symmetric space.

\section{A Character Approach.}

4.1. The Vanishing Theorem. In the course of the following three sections we prove the following theorem.

THEOREM 4.2. For the compact locally symmetric space $X=K \backslash G / \Gamma$ with $G$ locally isomorphic to $S O_{0}(2 n+1,1), K$ its maximal compact subgroup and $\Gamma$ a discrete torsion-free cocompact subgroup, the twisted torsion

$$
\left|\tau_{\theta}\right|=1
$$


for all outer automorphisms $\theta$ of $G$ with the property that $\theta^{2}=1$ and $\theta(K)=K$, $\theta(\Gamma)=\Gamma$.

Since in this case it suffices to consider only the Cartan involution for the automorphism $\theta$, and in view of the reduction to principal series, it is enough that we show

TheOREM 4.3. For the Cartan involution $\theta$, the twisted torsion of all $\theta$-invariant principal series representations $I(P, \sigma, \nu)=I$ of $G \cong S O_{0}(2 n+1,1)$

$$
\operatorname{tor}^{\theta}(I)=\left.\sum_{i}(-1)^{i} i \operatorname{tr} \theta\right|_{\operatorname{Hom}_{K}\left(\wedge^{i} \mathrm{p}, I\right)}=0 .
$$

We change our viewpoint by considering the disconnected group $\tilde{G}$ given by the semi-direct product $G \ltimes\{1, \theta\}$, with multiplication defined by

$$
\left(g_{1}, \theta^{i}\right) \cdot\left(g_{2}, \theta^{j}\right)=\left(g_{1} \theta^{i}\left(g_{2}\right), \theta^{i+j}\right) \quad \text { for } i, j \in\{1,2\} \text {. }
$$

This group has two components: the connected component of the identity which is clearly isomorphic to $G$, and a second component consisting of all elements of the form $(g, \theta)$ which we denote by $G \theta$.

We want to extend the representation $(I, \pi)$ of $G$ to a representation on $\tilde{G}$. There are two distinct ways to think about this extension. First, we can look at the induced representation $\operatorname{Ind}_{G}^{\bar{G}}(I)$. Since we have assumed that $I \cong I^{\theta}$, this induced representation will have two irreducible components, each corresponding to the choice of sign of the intertwining operator $A_{\theta}$. Second, we can define the extension of $I$ to $\tilde{G}$ by

$$
\pi\left(g \ltimes \theta^{i}\right)=\pi(g) A_{\theta}^{i} \quad \text { for } i \in\{1,2\} .
$$

Just as in [1],we make the following definition:

DEFINITION 4.4. The twisted character of the representation $(I, \pi)$ is the distribution on $G$ whose value on $f \in C_{c}^{\infty}(G)$ is given by

$$
\operatorname{trace}\left(\pi(f) A_{\theta}\right) \text {. }
$$

Hence, the twisted character is in fact the trace of the irreducible factor $\tilde{I}$ of the extension of $\operatorname{Ind}_{G}^{\tilde{G}}(I)$ on the component $G \theta$.

4.5. A Different View of the Twisted Torsion. In the disconnected group $\tilde{G}$ the analogue of the maximal compact subgroup $K$ of $G$ is the group $\tilde{K}=K \ltimes\{1, \theta\}$. For $G \cong S O_{0}(2 n+1,1)$ we have that

LEMMA 4.6. $\tilde{K}$ is isomorphic to the compact group $O(2 n+1)$, and thus is the direct product $S O(2 n+1) \times \mathbb{Z}_{2}$.

Proof. The disconnected group $\tilde{K}$ has its identity component isomorphic to $S O(2 n+1)$ and all $\tilde{k} \in \tilde{K}$ are orthogonal:

$$
(k, \theta) \cdot(k, \theta)^{t}=(k, \theta) \cdot\left(k^{t}, \theta\right)=\left(k \theta\left(k^{t}\right), 1\right)=\left(k k^{t}, 1\right)=1 .
$$

The second statement follows from [4].

Just as we can think of $\operatorname{dim} \operatorname{Hom}_{K}\left(\wedge^{i} \mathrm{p}, I\right)$ as the coefficient of the trivial representation of $K$ in the tensor product $\wedge^{i} \mathrm{p}^{*} \otimes I$, we can interpret $\left.\operatorname{tr} \theta\right|_{\operatorname{Hom}_{K}\left(\wedge^{i} \mathrm{p}, I\right)}$ as the coefficient of the trivial representation of $\tilde{K}$ when restricted to its $\theta$-component 
in the tensor product $\wedge^{i} \mathrm{p}^{*} \otimes \tilde{I}$. This is because $I$ extends to the $\theta$-component of $K$ precisely by the intertwining operator $A_{\theta}$ (see (4.1)). Hence, just as the usual torsion associated to the principal series $I$ is the coefficient of the trivial representation in the Grothendieck group of $K$ of the tensor product

$$
\sum_{i}(-1)^{i} i \wedge^{i} \mathrm{p}^{*} \otimes I
$$

(see [20]), we can interpret the twisted torsion $\operatorname{tor}^{\theta}(I)$ as the coefficient of the trivial representation in the Grothendieck group of $\tilde{K}$ when restricted to the component $K \theta$ of the tensor product

$$
\sum_{i}(-1)^{i} i \wedge^{i} \mathrm{p}^{*} \otimes \tilde{I}
$$

This means that to prove the vanishing theorem 4.3 it will suffice to calculate the virtual character of $K \theta$ on $\sum_{i}(-1)^{i} i \wedge^{i} \mathrm{p}^{*}$ and the twisted character $\operatorname{tr}\left(\left.\tilde{I}\right|_{G \theta}\right)$. Then, after restricting the latter to $K \theta$, we can multiply them together as they are characters of the non-identity component of the compact disconnected group $\tilde{K} \cong O(2 n+1)$.

4.7. Virtual Character on $\sum_{i}(-1)^{i} i \wedge^{i} \mathrm{p}^{*}$. Let $\mathfrak{t}$ denote a Cartan subalgebra of $\mathfrak{k}$. Denote the set of roots of $\mathfrak{t}$ on $p$ by $\sum(\mathfrak{t}, p)$. Here the dimension of the maximal abelian subspace $\mathfrak{a}$ of $p$ equals the multiplicity of the 0 -weight space, which is 1 in our case. Furthermore, if a root $\alpha$ is an element of $\sum(\mathfrak{t}, \mathrm{p})$, then so is $-\alpha$.

As "shown in [20], the character of $K$ of the virtual representation $\sum_{i}(-1)^{i} i \wedge^{i} \mathrm{p}^{*}=d E$ is given by $\left.\frac{d}{d t}\left(\rho_{E}(t)\right)\right|_{t=1}$ where $\rho_{E}(t)=\prod_{\alpha \in \sum(t, \mathrm{p})}\left(1-t e^{\alpha}\right)$. We use this result to obtain the character of $\tilde{K}$ restricted to the $\theta$-component.

Proposition 4.8. Define the function $\tilde{\rho}_{E}(t)=\prod_{\alpha \in \sum(t, p)}\left(1+t e^{\alpha}\right)$. Then $\left.\frac{d}{d t}\left(\tilde{\rho}_{E}(t)\right)\right|_{t=1}$ is the character of $\tilde{K}$ on the component $K \theta$ of the virtual representation $\sum_{i}(-1)^{i} i \wedge^{i} \mathrm{p}^{*}$.

Proof. It is shown in [20] that $\rho_{E}(1)=\prod_{e^{\alpha} \in \sum(T, \mathrm{p})}\left(1-e^{\alpha}\right)$, for $T$ a Cartan subgroup of $K$, is the character of $K$ on $\sum_{i}(-1)^{i} \wedge^{i} \mathrm{p}^{*}$. We extend this to the $\theta$ component of $\tilde{K}: \prod_{e^{\alpha} \in \sum(T \theta, \mathrm{p})}\left(1-e^{\alpha}\right)$, where $T \theta$ is the $\theta$-component of $\tilde{T}=T \ltimes\{1, \theta\}$ and $\sum(T \theta, \mathrm{p})=\left\{\alpha \in \mathfrak{t}^{*} \mid A d(t \theta) X=e^{\alpha\left(X_{t}\right)} X\right\}$ with $t=e^{X_{t}}$. If $e^{\alpha} \in \sum(T \theta, \mathrm{p})$, then $-e^{\alpha} \in \sum(T, p)$ and vice versa, since

$$
\operatorname{Ad}(t \theta) X_{t}=t \theta X_{t} \theta^{-1} t^{-1}=-\left(t X_{t} t^{-1}\right) .
$$

Therefore,

$$
\prod_{e^{\alpha} \in \sum(T, \mathrm{p})}\left(1+e^{\alpha}\right)=\prod_{e^{\alpha} \in \sum(T \theta, \mathrm{p})}\left(1-e^{\alpha}\right) .
$$

Finally, observe that the coefficient of $t^{i}$ in $\tilde{\rho}_{E}(t)$ is the character of $K \theta$ on $\wedge^{i} \mathrm{p}^{*}$ multiplied with $(-1)^{i}$, which proves the required result.

Lemma 4.9. For $S O_{0}(2 n+1,1)$ we have that

$$
\left.\frac{d}{d t}\left(\tilde{\rho}_{E}(t)\right)\right|_{t=1}=(2 n+1) \prod_{i=1}^{n}\left(1+e^{\alpha_{i}}\right)\left(1+e^{-\alpha_{i}}\right)
$$


for $\alpha_{1}, \ldots, \alpha_{n}$ in $\sum(\mathfrak{t}, \mathrm{p})$.

Proof. This is an application of the product rule and the identity

$$
e^{\alpha_{j}}\left(1+e^{-\alpha_{j}}\right)+e^{-\alpha_{j}}\left(1+e^{\alpha_{j}}\right)=\left(1+e^{\alpha_{j}}\right)\left(1+e^{-\alpha_{j}}\right)
$$

for all $1 \leq j \leq n$. as

REMARK. Note that we can rewrite this formula for the character $\frac{d}{d t}\left(\tilde{\rho}_{E}(t) \mid\right)_{t=1}$

$$
(2 n+1) \prod_{i=1}^{n}\left(e^{\alpha_{i} / 2}+e^{-\alpha_{i} / 2}\right)^{2}
$$

since $\left(1+e^{\alpha_{i}}\right)\left(1+e^{-\alpha_{i}}\right)=2+e^{\alpha_{i}}+e^{-\alpha_{i}}=\left(e^{\alpha_{i} / 2}+e^{-\alpha_{i} / 2}\right)^{2}$ for all $1 \leq i \leq n$.

4.10. The $\theta$-regular Elements in $S O_{0}(2 n+1,1)$. For the purposes of calculating the twisted character $\operatorname{tr}\left(\left.\tilde{I}\right|_{G \theta}\right)$ we need to extend the notion of regularity of elements to the group $\tilde{G}$. Following the presentation of Bouaziz in [3], for $x \in \tilde{G}$, we can look at $l(x)$, the multiplicity of the eigenvalue 1 of $A d(x)$. We put $l\left(\tilde{G}_{x}\right)=\inf _{y \in \tilde{G}_{x}} l(y)$ where $\tilde{G}_{x}$ denotes the connected component of $\tilde{G}$ containing the element $x$.

Definition 4.11. Consider the analytic function on $\tilde{G}$ given by

$$
\operatorname{det}(y+1-A d(x))=D(x) y^{l\left(\tilde{G}_{x}\right)} \quad \bmod \left(y^{l\left(\tilde{G}_{x}\right)+1}\right)
$$

where $y$ is a variable. An element $x \in \tilde{G}$ is regular if and only if $D(x) \neq 0$.

This definition allows us to conclude, just as in the connected case, that the set of regular elements $\tilde{G}^{\prime}$ of $\tilde{G}$ is an open $\tilde{G}$-invariant dense set in $\tilde{G}$.

In the case we are considering we are interested in the regular elements on the $\theta$-component $G \theta$ of $\tilde{G}$. Their counterparts in the component of the identity (under the obvious correspondence $(g, \theta) \mapsto g)$ are the elements we call the $\theta$-regular elements of $G \cong S O_{0}(2 n+1,1)$.

We follow the procedure described in Lemma 1.6.3 in [3] to find the conjugacy classes of regular elements in $G \theta$. Let $x=x\left(\alpha_{1}, \ldots, \alpha_{n}\right)$ for $0 \leq \alpha_{i} \leq 2 \pi$ be the element in the maximal torus $T$ of $K$ given by the following matrix:

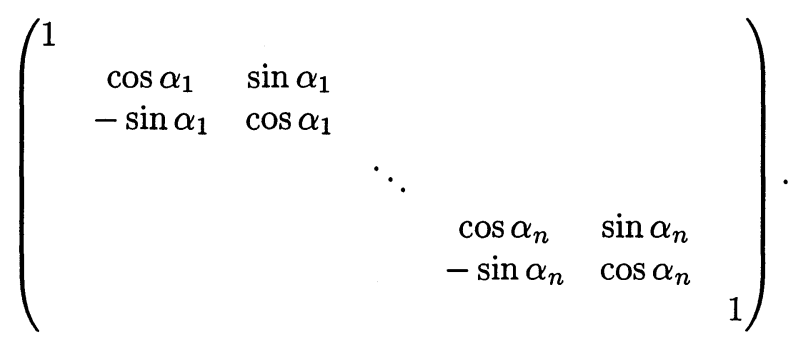

We first look at the algebra $\mathfrak{a}^{x \theta}=\{Y \in \mathfrak{g} \mid x \theta(Y)=Y x\}$. Then the group $H=$ $Z\left(G, \mathfrak{a}^{x \theta}\right)$ that consists of the set

$$
\left\{g \in S O_{0}(2 n+1,1) \mid g A=A g \text { for all } A \in \mathfrak{a}^{x \theta}\right\}
$$

has two connected components: the connected one containing the identity is precisely the maximal torus $T$ of $K$, and the other component is $-I d_{S O_{0}(2 n+1,1)} T$. Thus we 
already get that the elements of the form $h \theta$ for $h \in H$ and $\alpha_{i} \neq 0, \pi$ for all $1 \leq i \leq n$, as well as their conjugates under $G$, are regular elements on $G \theta$.

It is easy to verify that the only subalgebra of the form $\mathfrak{a}^{y \theta} \subseteq \mathfrak{h}=\mathfrak{a} \oplus \mathfrak{t}$, contained in the unique (up to conjugacy class) Cartan subalgebra $\mathfrak{h}$ of $G$, comes only from elements $y \in T$.

Finally, we have to determine whether there is more than one conjugacy class of $\theta$-Cartan subalgebras $\mathfrak{a}^{y \theta} \subset \mathfrak{h}$. By Lemma 1.6.3 in [3] any two subalgebras are conjugate via an element of the Weyl group $W\left(G_{\mathbb{C}}, \mathfrak{h}_{\mathbb{C}}\right)$. In our case this is the Weyl group for type $D_{n+1}$, which is isomorphic to $S_{n} \ltimes \mathbb{Z}_{2}^{n}$. If we think of the elements in $\mathfrak{h}$ as matrices $H(a, \bar{e})$ parametrized by $a \in \mathbb{R}$ and $\bar{e}=\left(e_{1}, \ldots, e_{n}\right) \in \mathbb{R}^{n}$, then we can exhibit an element $g \in S O(2 n+2, \mathbb{C})$ such that $g H(a, \bar{e}) g^{-1}$ is a diagonal matrix with $2 n$ imaginary eigenvalues. Then it becomes clear that every element of the Weyl group which takes $\mathfrak{h}$ into $\mathfrak{h}$ leaves $\mathfrak{a}^{x \theta}$ invariant. Therefore, we have shown that

Proposition 4.12. Every regular element in $G \theta$ is conjugate via an element of $G$ to an element of the form $t \theta$ or $-t \theta$ for $t=t\left(\alpha_{1}, \ldots, \alpha_{n}\right) \in T$, the maximal torus of $K$, with each $\alpha_{i} \neq 0, \pi$ for $1 \leq i \leq n$.

REMARK. Let us denote the $\theta$-Cartan subgroup $T \cup(-I d) T$ by $T_{G}$. Notice then that the above proposition says that we can write every $\theta$-regular element of $g$ of $G$ in the form $y t \theta(y)^{-1}$ for some $y \in G$ (since $\left.(g, \theta)=(y, 1)(t, \theta)\left(y^{-1}, 1\right)=\left(y t \theta(y)^{-1}, \theta\right)\right)$ and some $t \in T_{G}$ with each $i \neq 0, \pi$ for $1 \leq i \leq n\left(t \in T_{G}^{\prime}\right)$.

5. Calculation of the Twisted Character. Recall from section 4 that we are considering $\theta$-invariant principal series representations $I=(I(P, \sigma, \nu), \pi)$ of $G \cong$ $\mathrm{SO}_{0}(2 n+1,1)$.

Proposition 5.1. For a function $f \in C_{c}^{\infty}(G)$ we have that

$$
\operatorname{trace}\left(\pi(f) A_{\theta}\right)=\int_{K M A N} \epsilon_{\theta} f\left(k \operatorname{man} w_{0}^{-1} k^{-1}\right) e^{(\nu+\rho) \log a} \chi_{\sigma}(m) d m d a d n d k,
$$

with $\chi_{\sigma}$ denoting the character of the representation $\sigma$ of $M$.

Proof. We assume the conventions on fixing the Haar measures on $G, K, M$, $A$ and $N$ which appear in [12] and [13]. We follow the presentation of Chapter X, section 3 , in the latter source.

Using the decomposition $G=K M A N$ we can write an element $x$ of $G$ in a non-unique fashion as

$$
x=\kappa(x) \mu(x)(\exp H(x)) n .
$$

Then for the vector-valued function $\varphi \in L^{2}(K)$ taking its values in the Hilbert space $V^{\sigma}$, in the compact picture of the principal series representation, we have that

$$
\pi(x) A_{\theta}(\varphi(k))=e^{-(\nu+\rho) H\left(x^{-1} k w_{0}^{-1}\right)} \sigma\left(\mu\left(x^{-1} k w_{0}^{-1}\right)\right)^{-1} a_{\theta} \varphi\left(\kappa\left(x^{-1} k w_{0}^{-1}\right)\right),
$$

because $A_{\theta}(\varphi(k))=a_{\theta} \varphi\left(k w_{0}^{-1}\right)$. For the projection operator $E$ given by

$$
E \varphi(k)=\int_{K \cap M} \sigma(s) \varphi(k s) d s,
$$

we look at $\pi(f) E\left(A_{\theta} \varphi\right)(k)$ :

$$
\int_{G} e^{-(\nu+\rho) H\left(x^{-1} k w_{0}^{-1}\right)} \sigma\left(\mu\left(x^{-1} k w_{0}^{-1}\right)\right)^{-1} f(x) E\left(a_{\theta} \varphi\right)\left(\kappa\left(x^{-1} k w_{0}^{-1}\right)\right) d x .
$$


The change of variables $x k w_{0}^{-1} \mapsto x$ of the unimodular group $G$ followed by 8.44 in

[12] produces then

$$
\begin{array}{r}
\int_{K \times M \times A \times N} e^{-(\nu-\rho) \log a} \sigma(m)^{-1} f\left(k w_{0}^{-1} n^{-1} a^{-1} m^{-1} k^{\prime-1}\right) \\
\times E\left(a_{\theta} \varphi\left(k^{\prime}\right)\right) d k d m d a d n \\
\end{array}
$$

Now we substitute the formula for $E$ as above. After we perform the change of variables $k^{\prime} s \mapsto k^{\prime}$ and observe that, by the normalization of the Haar measure on $K \cap M$, the integral there is 1 , we obtain

$$
\int_{K \times M A N} e^{(\nu-\rho) \log a^{-1}} \sigma\left(m^{-1}\right) f\left(k w_{0}^{-1}(\operatorname{man})^{-1} k^{\prime-1}\right) a_{\theta} \varphi\left(k^{\prime}\right) d k^{\prime} d_{l}(\operatorname{man}) .
$$

By 8.30 in [12] this can be written in the form

$$
\int_{K \times(M A N)} e^{(\nu+\rho) \log a} \sigma(m) f\left(k w_{0}^{-1} m a n k^{\prime-1}\right) a_{\theta} \varphi\left(k^{\prime}\right) d k^{\prime} d m d a d n .
$$

Since the expression

$$
\int_{M A N} f\left(k w_{0}^{-1} \operatorname{mank}^{-1}\right) e^{(\nu+\rho) \log a} \sigma(m) d m d a d n
$$

just as in [13] is a smooth compact average of a trace class operator, and as $\chi_{\sigma}$ exists and $\pi(f) E$ is of trace class, then by Lemma 10.15 in the same source, we can conclude that

$$
\operatorname{trace}\left(\pi(f) A_{\theta}\right)=\int_{K M A N} \epsilon_{\theta} f\left(k w_{0}^{-1} \operatorname{mank}^{-1}\right) e^{(\nu+\rho) \log a} \chi_{\sigma}(m) d m d a d n d k
$$

with $\epsilon_{\theta}$ the choice of sign coming from $a_{\theta}$. Now the change of variables $k w_{0}^{-1} \mapsto k$ gives the desired result.

Before we proceed with the calculation of the trace, we need an analogue of the Weyl Integral Formula (see p. 141 in [13]) for the twisted case.

LemmA 5.2. The map $\Phi: G / T_{G} \times T_{G}^{\prime} \rightarrow \bigcup_{x \in G} x T_{G}^{\prime} \theta(x)^{-1}$ given by

$$
(x, t) \mapsto x t \theta(x)^{-1}
$$

is an everywhere regular ||$W\left(G, T_{G}\right)|: 1|$ map, with $W\left(G, T_{G}\right)$ denoting the Weyl group $N_{G}\left(T_{G}\right) / Z_{G}\left(T_{G}\right)$.

Proof. Recall that the elements of $T_{G}$, as determined in the previous section, are fixed by the automorphism $\theta$.

Take a point $y=x t \theta(x)^{-1} \in \bigcup_{x \in G} x T_{G}^{\prime} \theta(x)^{-1}$. It is clear that the complete inverse image under $\Phi$ of $y$ consists of $\left|W\left(G, T_{G}\right)\right|$ points $(w \cdot x, w \cdot t)$ where $w \cdot x=$ $x t_{w} T, w \cdot t=t_{w} t \theta\left(t_{w}^{-1}\right)=t_{w} t t_{w}^{-1}$ for $t_{w} \in N_{G}\left(T_{G}\right)$, which are all distinct.

To show regularity of $\Phi$ we follow the proof of Proposition 1.4.2.3 in [25]. A calculation for $p_{0}=\left(x_{0} T_{G}, t_{0}\right) \in G / T_{G} \times T_{G}^{\prime}$ then produces that

$$
\left|\operatorname{det}(d \Phi)_{p_{0}}\right|=\left|\operatorname{det}\left(A d\left(t_{0}\right)^{-1}-\theta\right)_{\mathfrak{g} / \mathfrak{t}}\right| \text {. }
$$


But the latter term equals

$$
\left|\operatorname{det}\left(A d\left(t_{0}\right)^{-1} \circ \theta-1\right)_{\mathfrak{g} / \mathfrak{t}}\right|
$$

since the automorphism $\theta^{2}=1$. We chose $t_{0} \in T_{G}^{\prime}$, so by the observation on regularity following equation 4.11 , we have that

$$
\left|\operatorname{det}(d \Phi)_{p_{0}}\right| \neq 0
$$

and so $\Phi$ is regular.

We know from section (4.10) that the $\theta$-regular elements of $G$ form a dense subset and each of them can be expressed as a union of $\theta$-conjugates $g t \theta(g)^{-1}$ of $T_{G}^{\prime}$. Therefore, if we normalize the Haar measures in such a way that for $f \in C_{c}^{\infty}(G)$

$$
\int_{G} f(x) d_{G}(x)=\int_{G / T_{G}}\left\{\int_{T_{G}} f(x t) d_{T_{G}} t\right\} d_{G / T_{G}} \bar{x},
$$

we obtain the twisted version of the Weyl Integral Formula:

$$
\begin{aligned}
\int_{G} f(x) d_{G}(x)= & \left|W\left(G, T_{G}\right)\right|^{-1} \\
& \times \int_{T_{G}}\left|\operatorname{det}\left(A d(t)^{-1} \circ \theta-1\right)\right|_{\mathfrak{g} / \mathfrak{t}} d_{T_{G}} t \\
& \times \int_{G / T_{G}} f\left(x t \theta(x)^{-1}\right) d_{G / T_{G}} \bar{x} .
\end{aligned}
$$

To proceed with the derivation of the formula for $\operatorname{trace}\left(\pi(f) A_{\theta}\right)$ we are going to apply the twisted version of the Weyl Integral Formula to the reductive group $M A$. First, observe that $T_{G}$ is still a $\theta$-Cartan subgroup in this case. Let us write an element $t \in T_{G}$ as $t=t_{A} t_{M}$ with $t_{A} \in A, t_{M} \in M$. Note that $t_{A}$ can be either the identity or the element given by the diagonal matrix where the top-most and bottom-most entries are (-1)'s and the rest are 1's. In fact, the Weyl group $W\left(M A, T_{G}\right)=W\left(M, T_{G}\right)=W(M, T)$ with $T$ the Cartan subgroup of $K$ and of $M$, since $a t a^{-1}=t$ for all $a \in A, t \in T_{G}\left(\right.$ as $\mathfrak{t} \subset \mathfrak{m}=Z_{\mathfrak{k}(\mathfrak{a})}$ and $\left.T_{G} \cong T \times \mathbb{Z}_{2}\right)$. Moreover, we have that

$$
\left|\operatorname{det}\left(A d(t)^{-1} \circ \theta-1\right)\right|_{(\mathfrak{m} \oplus \mathfrak{a}) / \mathfrak{t}}=2\left|\operatorname{det}\left(A d\left(t_{M}\right)^{-1}-1\right)\right|_{\mathfrak{m} / \mathfrak{t}}
$$

because $\theta(m)=m$ for all $m \in M$, and $A d(t)^{-1} \theta(Y)-Y=-2 Y$ for all $Y \in \mathfrak{a}$. Furthermore, we have that

$$
\left|\operatorname{det}\left(A d\left(t_{M}\right)^{-1}-1\right)\right|_{\mathfrak{m} / \mathfrak{t}}=\left|\Delta_{M}\left(t_{M}\right)\right|^{2},
$$

where following the notation in [26],

$$
\Delta_{M}\left(t_{M}\right)=\xi_{\rho_{M}}\left(t_{M}\right) \prod_{\alpha \in \Delta^{+}\left(\mathfrak{t}_{\mathbb{C}}, \mathfrak{m}_{\mathbb{C}}\right)}\left(1-\xi_{\alpha}\left(t_{M}\right)^{-1}\right)
$$

for the positive roots $\alpha$, their exponentials $\xi_{\alpha}$, and their half-sum $\rho_{M}$. 
We begin by applying the Twisted Weyl Integral Formula to the group $M A$ in the trace formula (5.1):

$$
\begin{aligned}
\operatorname{trace}\left(\pi(f) A_{\theta}\right)=2 \epsilon_{\theta}|W(M, T)|^{-1} \int_{T}\left|\Delta_{M}\left(t_{M}\right)\right|^{2} \chi_{\sigma}\left(t_{M}\right) d t \\
\times \int_{K} \int_{M A / T} \int_{N} f\left(k m a t \theta(m a)^{-1} n w_{0}^{-1} k^{-1}\right) d k d n d(\overline{m a}) .
\end{aligned}
$$

LEMMA 5.3. Let $h \in M A$ be such that $\operatorname{det}\left(A d(h)^{-1}-\theta\right)_{\mid \operatorname{diag}(\mathfrak{n} \oplus \bar{n})} \neq 0$, with $\overline{\mathfrak{n}}=\theta(\mathfrak{n})$ and $\operatorname{diag}(\mathfrak{n} \oplus \overline{\mathfrak{n}})=\{(Z, \theta(Z)) \mid Z \in \mathfrak{n}\}$. Then the mapping $\xi$ defined by

$$
(n, \theta(n)) \mapsto h^{-1} w_{0} \theta(n) h^{w_{0}^{-1}} n^{-1}
$$

is an analytic diffeomorphism of $\operatorname{diag}(N \times \theta(N))$ onto $w_{0} \operatorname{diag}(\theta(N) N)$.

Proof. It is clear that $\xi$ is analytic. Given $h^{w_{0}^{-1}}=w_{0}^{-1} h w_{0}$, and fixing $n \in N$, $Z \in \mathfrak{n}$, we have that

$$
\begin{aligned}
& \xi(n \exp (t Z), \theta(n) \exp (t \theta(Z)))= \\
& \xi(n, \theta(n)) \exp \left(t A d\left(n\left(h^{w_{0}^{-1}}\right)^{-1}\right) \theta(Z)\right) \exp (-t A d(n) Z) .
\end{aligned}
$$

Therefore, since $\theta^{w_{0}^{-1}}=\theta$, we get that

$$
\operatorname{det}(d \xi)_{n}=\operatorname{det}\left(A d\left(h^{w_{0}^{-1}}\right)^{-1} \circ \theta-1\right)_{\mid \operatorname{diag}(\mathfrak{n} \oplus \overline{\mathfrak{n}})}=\operatorname{det}\left(A d(h)^{-1}-\theta\right)_{\mid \operatorname{diag}(\mathfrak{n} \oplus \overline{\mathfrak{n}})} \neq 0,
$$

which implies that $\xi$ is everywhere regular.

An inductive argument with $N$ then shows that $\xi$ is in fact $1-1$ and onto (see Lemma 10.16 in [13]).

Corollary 5.4. Fix $h \in M A$ such that $\operatorname{det}\left(A d(h)^{-1}-\theta\right)_{\mid \operatorname{diag}(\mathfrak{n} \oplus \overline{\mathfrak{n}})} \neq 0$. Then

$$
\begin{aligned}
& \int_{w_{0} N} f\left(w_{0} \theta(n) h^{w_{0}^{-1}} n^{-1} w_{0}^{-1}\right) d\left(w_{0} n\right) \\
& =\left|\operatorname{det}\left(A d(h)^{-1}-\theta\right)\right|_{\operatorname{diag}(\mathfrak{n} \oplus \overline{\mathfrak{n}})}^{-1} \int_{N} f\left(h n w_{0}^{-1}\right) d n
\end{aligned}
$$

for any function $f \in C_{c}^{\infty}(G)$.

We apply lemma 5.3 to the element $h=\operatorname{mat} \theta(m a)^{-1}$ of $M A$. Then

$$
\left(A d\left(m a t \theta(m a)^{-1}\right)-\theta\right)_{\mid \operatorname{diag}(\mathfrak{n} \oplus \overline{\mathfrak{n}})}=\left(A d\left(t^{-1}\right)-\theta\right)_{\mid \operatorname{diag}(\mathfrak{n} \oplus \overline{\mathfrak{n}})}
$$

since $\operatorname{det} A d(\theta(\tilde{m})) \operatorname{det} A d(\tilde{m})^{-1}=1$ for $\tilde{m} \in M A$. Therefore, we have that

$$
\left|\operatorname{det}\left(A d(t)^{-1}-\theta\right)\right|_{\operatorname{diag}(\mathfrak{n} \oplus \overline{\mathfrak{n}})}=s^{N / T} \prod_{\alpha \in \Delta+\left(\mathfrak{t}_{\mathfrak{c}}, \operatorname{diag}\left(\mathfrak{n}_{\mathfrak{c}} \oplus \overline{\mathfrak{n}}_{\mathbb{C}}\right)\right)}\left(1-\xi_{\alpha}(t)^{-1}\right)
$$

with $s^{N / T}=(-1)^{\text {number of roots in } \Delta^{+}\left(\mathfrak{t}_{\mathbb{C}}, \operatorname{diag}\left(\mathbf{n}_{\mathbb{C}} \oplus \overline{\mathbf{n}}_{\mathbb{C}}\right)\right)}$.

Now let us substitute this corollary of lemma 5.3 in the equation (5.7): 
$(5.9)$

$$
\begin{gathered}
\operatorname{trace}\left(\pi(f) A_{\theta}\right)=2 \epsilon_{\theta}|W(M, T)|^{-1} \\
\times \int_{T_{G}}\left|\Delta_{M}\left(t_{M}\right)\right|^{2} \chi_{\sigma}\left(t_{M}\right)\left|\operatorname{det}\left(A d(t)^{-1}-\theta\right)\right|_{\operatorname{diag}(\mathfrak{n} \oplus \overline{\mathfrak{n}})} d t \\
\times \int_{K} \int_{M A / T} \int_{w_{0} N} f\left(k w_{0} \theta(n) w_{0}^{-1} \operatorname{mat} \theta(m a)^{-1} w_{0} n^{-1} w_{0}^{-1} k^{-1}\right) d k d\left(w_{0} n\right) d \overline{m a} .
\end{gathered}
$$

We do a change of variables $w_{0} n \mapsto n$. The observation that $\theta(n)=w_{0}^{-1} n w_{0}$ and the change of variables $k w_{0}^{-1} \mapsto k$ let us rewrite the triple integral in the following form:

$$
\int_{K} \int_{N} \int_{M A / T} f\left(k n m a t \theta(m a)^{-1} \theta(n)^{-1} k^{-1}\right) d k d n d \overline{m a} .
$$

Putting it all together then we get:

$$
\begin{aligned}
& \operatorname{trace}\left(\pi(f) A_{\theta}\right)=2 \epsilon_{\theta}|W(M, T)|^{-1} \\
& \quad \times \int_{T_{G}}\left|\Delta_{M}\left(t_{M}\right)\right|^{2} \chi_{\sigma}\left(t_{M}\right) s^{N / T} \prod_{\alpha \in \Delta^{+}\left(\mathfrak{t}_{\mathbb{C}}, \operatorname{diag}\left(\mathfrak{n}_{\mathbb{C}} \oplus \tilde{\mathfrak{n}}_{\mathbb{C}}\right)\right)}\left(1-\xi_{\alpha}(t)^{-1}\right) d t \\
& \times \int_{G^{\prime} T_{G}} f_{\theta}\left(g t \theta(g)^{-1}\right) d \bar{g}
\end{aligned}
$$

where $f_{\theta}\left(g t g^{-1}\right)=f\left(k n m a t \theta(k n m a)^{-1}\right)$, since $\theta(k)=k$ for all $k \in K$.

A direct calculation shows that

$$
s^{N / T} \prod_{\alpha \in \Delta^{+}\left(\mathfrak{t}_{\mathbb{C}}, \operatorname{diag}\left(\mathfrak{n}_{\mathbb{C}} \oplus \overline{\mathbf{n}}_{\mathbb{C}}\right)\right)}\left(1-\xi_{\alpha}(t)^{-1}\right)=s^{K / M} \prod_{i=1}^{n}\left(1-\xi_{\alpha_{i}}\left(t_{M}\right)^{-1}\right)
$$

for $\alpha_{1}, \ldots, \alpha_{n}$ the positive roots for $S O_{0}(2 n+1,1)$ in $\Delta^{+}\left(\mathfrak{t}_{\mathbb{C}}, \mathfrak{k}_{\mathbb{C}}\right)$ which are not in $\Delta^{+}\left(\mathfrak{t}_{\mathbb{C}}, \mathfrak{m}_{\mathbb{C}}\right)$, and $s^{K / M}$ again denoting $(-1)$ to the power equaling the number of positive roots in this product. To keep the notation consistent, set

$$
\xi_{\frac{\alpha_{1}+\cdots+\alpha_{n}}{2}}=: \xi_{\rho_{K / M}}
$$

and

$$
\xi_{\rho_{K / M}}\left(t_{M}\right) \prod_{i=1}^{n}\left(1-\xi_{\alpha_{i}}\left(t_{M}\right)^{-1}\right)=: \Delta_{K / M}\left(t_{M}\right) .
$$

Then we have the following equality:

$$
s^{G / T_{G}} \Delta^{\theta}(t)=s^{M / T} \Delta_{M}\left(t_{M}\right) \sqrt{2} s^{K / M} \Delta_{K / M}\left(t_{M}\right)
$$

with

$$
\left|\operatorname{det}\left(A d(t)^{-1} \circ \theta-1\right)\right|_{\mathfrak{g} / \mathfrak{t}}=\left|\Delta^{\theta}(t)\right|^{2} .
$$


By analogy with the usual invariant integral, consider

$$
\Phi_{f_{\theta}}=\Delta^{\theta}(t) \int_{G / T_{G}} f_{\theta}\left(g t \theta(g)^{-1}\right) .
$$

Then the trace from equation (5.11) becomes

$$
\sqrt{2} \epsilon_{\theta}|W(M, T)|^{-1} \int_{T_{G}} s^{G / T} s^{M / T} \Delta_{M}\left(t_{M}\right) \chi_{\sigma}(t) \Phi_{f_{\theta}}(t) d t
$$

The integral $\Phi_{f_{\theta}}$ is invariant under conjugation by elements of $W(M, T)$ and so is $\chi_{\sigma}$. Moreover the quotient

$$
\frac{\xi_{\rho_{K / M}} \xi_{-\rho} \xi_{\rho_{M}} s^{G / T} \Delta^{\theta}}{s^{M / T} \Delta_{M}}=s^{K / M} \Delta_{K / M}
$$

is invariant under conjugation by all of $W\left(G, T_{G}\right)$. Therefore we can rewrite equation (5.13) as

$$
\begin{aligned}
& s^{G / T}\left|W\left(G, T_{G}\right)\right|^{-1} \\
& \quad \times \int_{T_{G}} \Phi_{f_{\theta}}(t)\left\{\frac{\epsilon_{\theta} s^{M / T} s^{G / T_{G}} \sum_{w \in W\left(G, T_{G}\right) / W(M, T)} \chi_{w \sigma}\left(t_{M}\right)}{\Delta_{K / M}\left(t_{M}\right)}\right\} \Delta^{\theta}(t) d t
\end{aligned}
$$

Hence, we have the following

THEOREM 5.5. The twisted character trace $\left(\pi(f) A_{\theta}\right)$ is a locally integrable function given by

$$
\frac{\epsilon_{\theta} s^{M / T} s^{G / T_{G}} \sum_{w \in W\left(G, T_{G}\right) / W(M, T)} \chi_{w \sigma}\left(t_{M}\right)}{\Delta_{K / M}\left(t_{M}\right)}
$$

on the $\theta$-regular elements of $G$ and 0 elsewhere.

Proof. Consider the function $F_{\theta}^{\sigma}$ on $G$ which vanishes outside the set of $\theta$-regular elements of the group and such that

1. $F_{\theta}^{\sigma}\left(x t \theta(x)^{-1}\right)=F_{\theta}^{\sigma}(t) \quad\left(t \in T_{G}, g \in G\right)$;

2. For $t \in T_{G}$

$$
F_{\theta}^{\sigma}(t)=\frac{\epsilon_{\theta} s^{M / T} s^{G / T_{G}} \sum_{w \in W\left(G, T_{G}\right) / W(M, T)} \chi_{w \sigma}\left(t_{M}\right)}{\Delta_{K / M}\left(t_{M}\right)} .
$$

We claim that, in the sense of distribution theory, $\operatorname{trace}\left(\pi(f) A_{\theta}\right)=F_{\theta}^{\sigma}$, for $f \in$ $C_{c}^{\infty}(G)$. By using the Twisted Weyl Integral Formula and the expression for $F_{\theta}^{\sigma}$ we obtain

$$
\begin{aligned}
& \int_{G} f(x) F_{\theta}^{\sigma}(x) d x \\
= & \left|W\left(G, T_{G}\right)\right|^{-1} \int_{T_{G}}\left|\Delta^{\theta}(t)\right|^{2} F_{\theta}^{\sigma}(t) d t \int_{G / T_{G}} f\left(g t \theta(g)^{-1}\right) d \bar{g} \\
= & s^{G / T_{G}}\left|W\left(G, T_{G}\right)\right|^{-1} \int_{T_{G}} \Delta^{\theta}(t) F_{\theta}^{\sigma}(t) \Phi_{f_{\theta}}(t) d t \\
= & \sqrt{2} \epsilon_{\theta}|W(M, T)|^{-1} \int_{T_{G}} s^{G / T_{G}} s^{M / T} \Delta_{M}\left(t_{M}\right) \chi_{\sigma}(t) \Phi_{f_{\theta}}(t) d t .
\end{aligned}
$$

This final equation is exactly what we had in (5.13). The local integrability follows from section 11.6 in [13]. 
6. Conclusion. We first rewrite the character formula $\left.\operatorname{tr} \tilde{I}\right|_{G \theta}$ for the groups $S O_{0}(2 n+1,1) \ltimes\{1, \theta\}$. Recall that the group $M$ here is isomorphic to $S O(2 n)$ and $T$ is its maximal torus. Thus we are in the situation of type $D_{n}$ and hence $|W(M, T)|=n !(2)^{n-1}$. Also, notice that we have in this case $s^{M / T}=1$. Furthermore, observe that $W\left(G, T_{G}\right)=W(K, T)$ and thus we are in the situation of type $B_{n}$. Then $\left|W\left(G, T_{G}\right)\right|=n ! 2^{n}$, so

$$
\left|W\left(G, T_{G}\right) / W(M, T)\right|=2 .
$$

In fact, we can choose the element $w_{0}$ as the nontrivial representative of this quotient. For $n$ odd we have $s^{G / T}=-1$, and for $n$ even $s^{G / T}=1$.

This allows us to rewrite the numerator of the twisted character formula in the following way:

$$
\sum_{w \in W\left(G, T_{G}\right) / W(M, T)} \chi_{w \sigma}(t)=\chi_{\sigma}(t)+\chi_{w_{0} \sigma}(t) .
$$

The class of representations of $M$ under consideration (see 3.2 in Chapter 2) are those for which $\sigma \cong w_{0} \sigma$; in fact it is the set given by the $n$ irreducible representations

$$
\mathcal{M}_{\sigma}=\left\{T_{t r}, V_{s t}, \wedge^{i} V_{s t} \mid 2 \leq i \leq n-1\right\} .
$$

Therefore, for the choice of $\epsilon_{\theta}=1$ we obtain the following form of the twisted character formula:

$$
\frac{(-1)^{n} 2 \chi_{\sigma}}{\Delta_{K / M}(t)} \quad \text { for } \sigma \in \mathcal{M}_{\sigma} .
$$

We would like to write the characters $\chi_{V_{s t}}, \ldots, \chi_{\wedge^{n-1} V_{s t}}$ in a suitable form. Let us denote the highest weight of the irreducible representation $\wedge^{k} V_{s t}$ by $\lambda=\alpha_{1}+\cdots+\alpha_{k}$, for $1 \leq k \leq n-1$. We use the combinatorial results described on p. 469 in [8]. It turns out that $\chi_{\wedge^{k} V_{s t}}$ equals the determinant of the following matrix

$$
\left(\begin{array}{cccccccccc}
h_{1} & h_{2} & h_{3} & \ldots & h_{k-1} & h_{k} & h_{k+1} & \ldots & h_{n-1} & h_{n} \\
1 & h_{1} & h_{2} & \ldots & h_{k-2} & h_{k-1} & h_{k} & \ldots & h_{n-2} & h_{n-1} \\
0 & 1 & h_{1} & \ldots & h_{k-3} & h_{k-2} & h_{k-1} & \ldots & h_{n-3} & h_{n-2} \\
\vdots & \vdots & \vdots & \ldots & \vdots & \vdots & \vdots & \ldots & \vdots & \vdots \\
0 & 0 & 0 & \ldots & 1 & h_{1} & h_{2} & \ldots & h_{n-k} & h_{n-k+1} \\
0 & 0 & 0 & \ldots & 0 & 1 & h_{1} & \ldots & h_{n-k-2} & h_{n-k-1} \\
\vdots & \vdots & \vdots & \ldots & \vdots & \vdots & \vdots & \ldots & \vdots & \vdots \\
0 & 0 & 0 & \ldots & 0 & 0 & 0 & \ldots & 1 & h_{1} \\
0 & 0 & 0 & \ldots & 0 & 0 & 0 & \ldots & 0 & 1
\end{array}\right) .
$$

Here $h_{i}$ 's are the complete symmetric polynomials of degree $i$ in the set of variables $\left\{z_{1}, \ldots, z_{n}, z_{1}^{-1}, \ldots, z_{n}^{-1}\right\}$ where $z_{i}=e^{\alpha_{i}}$. By expanding along the last $n-k$ rows, we conclude that this determinant is equal to the determinant of the $k \times k$ matrix:

$$
A_{k}=\left(\begin{array}{ccccc}
h_{1} & h_{2} & \ldots & h_{k-1} & h_{k} \\
1 & h_{1} & \ldots & h_{k-2} & h_{k-1} \\
0 & 1 & \ldots & h_{k-3} & h_{k-2} \\
\vdots & \vdots & \ldots & \vdots & \vdots \\
0 & 0 & \ldots & 1 & h_{1}
\end{array}\right) .
$$


THEOREM 6.1. We have $\operatorname{det} A_{k}=e_{k}$ for $1 \leq k \leq n-1$, where $e_{k}$ denotes the elementary symmetric polynomial of degree $k$ in the ordered set of variables $\left\{z_{1}, \ldots, z_{n}, z_{1}^{-1}, \ldots, z_{n}^{-1}\right\}$.

Proof. We prove this theorem by induction on $k$.

For $k=1$, we have that $h_{1}=e_{1}$. Let us assume now that $\operatorname{det} A_{l}=e_{l}$ for all positive integers $l \leq k-1$. Consider now $\operatorname{det} A_{k}$. We expand along the last row of $A_{k}$ to get

$$
\operatorname{det} A_{k}=h_{1} \operatorname{det} A_{k-1}-\operatorname{det} B_{k-1}\left(h_{2}\right) \text {, }
$$

with $B_{k-1}\left(h_{2}\right)$ denoting the $(k-1) \times(k-1)$ matrix

$$
B_{k-1}\left(h_{2}\right)=\left(\begin{array}{ccccc}
h_{1} & h_{2} & \ldots & h_{k-2} & h_{k} \\
1 & h_{1} & \ldots & h_{k-3} & h_{k-1} \\
0 & 1 & \ldots & h_{k-4} & h_{k-2} \\
\vdots & \vdots & \ldots & \vdots & \vdots \\
0 & 0 & \ldots & 1 & h_{2}
\end{array}\right) .
$$

By the induction hypothesis we have that $\operatorname{det} A_{k-1}=e_{k-1}$. Now expand $B_{k-1}\left(h_{2}\right)$ along the last row to get:

$$
\operatorname{det} B_{k-1}\left(h_{2}\right)=h_{2} \operatorname{det} A_{k-2}-\operatorname{det} B_{k-2}\left(h_{3}\right) .
$$

Now continuing this process, while successfully applying the induction hypothesis, we obtain the following equality:

$$
\operatorname{det} A_{k}=h_{1} e_{k-1}-h_{2} e_{k-2}+h_{3} e_{k-1}-\cdots+(-1)^{k} h_{k-3}+(-1)^{k+1} \operatorname{det} B_{3}\left(h_{k-2}\right) .
$$

Here

$$
\operatorname{det} B_{3}\left(h_{k-2}\right)=\left|\begin{array}{ccc}
h_{1} & h_{2} & h_{k} \\
1 & h_{1} & h_{k-1} \\
0 & 1 & h_{k-2}
\end{array}\right|=h_{k}+h_{1}^{2} h_{k-2}-h_{2} h_{k-2}-e_{1} h_{k-1} .
$$

We make use of Newton's identity (for example see [15]):

$$
h_{k}-e_{1} h_{k-1}+e_{2} h_{k-2}-\cdots+(-1)^{k} e_{k}=0 .
$$

Thus by plugging in the expression for $h_{k}=e_{1} h_{k-1}-e_{2} h_{k-2}+\cdots+(-1)^{k} e_{k}$ and $h_{2}=e_{1}^{2}-e_{2}$ in $\operatorname{det} B_{3}\left(h_{k-2}\right)$ we have that

$$
\begin{aligned}
& \operatorname{det} A_{k} \\
& \qquad \begin{aligned}
= & e_{k-1} h_{1}-e_{k-2} h_{2}+e_{k-3} h_{3}-\cdots+(-1)^{k} e_{3} h_{k-3} \\
& +(-1)^{k+1}\left(e_{3} h_{k-3}-e_{4} h_{k-4}+\cdots+(-1)^{k+1} e_{k}\right) \\
& =e_{k} .
\end{aligned}
\end{aligned}
$$

Definition 6.2. Let $i \leq n-1$. Then we define $S_{i}$ to be

$$
\sum_{1 \leq j_{1}<\cdots<j_{i} \leq n}\left(z_{j_{1}}+z_{j_{1}}^{-1}\right) \ldots\left(z_{j_{i}}+z_{j_{i}}^{-1}\right)
$$


where the sum is taken over all increasing sequences $1 \leq j_{1}<\cdots<j_{i} \leq n$ of length $i$ of integers between 1 and $n$.

THEOREM 6.3. We have the following expressions for the elementary symmetric polynomials $e_{k}$ in the variables $z_{j}, z_{j}^{-1}$ where $1 \leq j \leq n$ :

$$
\begin{array}{ll}
e_{k}=\sum_{i=0}^{j}\left(\begin{array}{c}
n-2 i-1 \\
j-i
\end{array}\right) S_{2 i+1} & \text { if } k=2 j+1 \\
e_{k}=\sum_{i=0}^{j}\left(\begin{array}{c}
n-2 i \\
j-i
\end{array}\right) S_{2 i} & \text { if } k=2 j .
\end{array}
$$

Proof. Consider the space of the elementary symmetric polynomials $e_{k}$ 's as above and the space of weighted sums of $S_{i}$ 's as above. It suffices to show that the dimensions of these two spaces are equal. In fact, the equality of these two numbers follows from a generating function argument, as follows.

LEMMA 6.4. Let $n$ and $k$ be positive integers with $k \leq n-1$. Then

$$
\begin{array}{ll}
\left(\begin{array}{c}
2 n \\
k
\end{array}\right)=\sum_{i=0}^{j} 2^{2 i+1}\left(\begin{array}{c}
n-2 i-1 \\
j-i
\end{array}\right)\left(\begin{array}{c}
n \\
2 i+1
\end{array}\right), & \text { if } k=2 j+1 \\
\left(\begin{array}{c}
2 n \\
k
\end{array}\right)=\sum_{i=0}^{j} 2^{2 i}\left(\begin{array}{c}
n-2 i \\
j-i
\end{array}\right)\left(\begin{array}{c}
n \\
2 i
\end{array}\right) & \text { if } k=2 j .
\end{array}
$$

Proof. Consider the generating function $(1+z)^{2 n}=\left(1+2 z+z^{2}\right)^{n}$ and compute the coefficient of $z^{k}$ on both sides.

This proves the theorem.

Let us conclude the proof of the vanishing theorem by considering first the case of the trivial representation of $M$. Its character equals 1 , so with the choice of $\epsilon_{\theta}=1$, the twisted character has the form:

$$
\frac{2(-1)^{n}}{\Delta_{K / M}}=\frac{2(-1)^{n}}{\prod_{i=1}^{n}\left(e^{\alpha_{i} / 2}-e^{-\alpha_{i} / 2}\right)} .
$$

The character formula of $K \theta$ on $\sum_{i}(-1)^{i} i \wedge^{i} \mathrm{p}^{*}$ is given by:

$$
(2 n+1) \prod_{i=1}^{n}\left(e^{\alpha_{i} / 2}+e^{-\alpha_{i} / 2}\right)^{2} .
$$

Hence we can write the product of the two characters in the following way:

$$
2(2 n+1)(-1)^{n} \prod_{i=1}^{n}\left(\frac{e^{\alpha_{i} / 2}+e^{-\alpha_{i} / 2}}{e^{\alpha_{i} / 2}-e^{-\alpha_{i} / 2}}\right)\left(e^{\alpha_{i} / 2}+e^{-\alpha_{i} / 2}\right) .
$$

For each $1 \leq i \leq n$, we can interpret the term

$$
\frac{e^{\alpha_{i} / 2}+e^{-\alpha_{i} / 2}}{e^{\alpha_{i} / 2}-e^{-\alpha_{i} / 2}}
$$


as the difference of the characters $\Theta_{\mathcal{D}_{2}^{+}}, \Theta_{\mathcal{D}_{2}^{-}}$of the two discrete series representations $\mathcal{D}_{2}^{+}$and $\mathcal{D}_{2}^{-}$of $S L(2, \mathbb{R})$. Now we work with $S O(2)$-types. On the right side of (6.7) we are left with terms of the form

$$
e^{\alpha_{i} / 2}+e^{-\alpha_{i} / 2}
$$

which correspond to the sum of the characters of the two irreducible representations parametrized by the integers 1 and -1 . However, on the left the $S O(2)$-types which appear (all with multiplicity 1 ) are the representations parametrized by $2,4,6, \ldots$ and $-2,-4,-6, \ldots$ Thus, we obtain that the coefficient of the trivial representation is 0 , for each $i$. Therefore, we have that the coefficient of the trivial representation is 0 in the whole product (6.7).

Next let us look at $\chi_{\wedge^{k} V_{s t}}$ for $1 \leq k \leq n-1$. We assume that $k$ is even (the case when $k$ is odd is handled completely analogously). Write $k=2 l$ for some positive integer $l$. We showed that

$$
\chi_{\wedge^{k} V_{s t}}=e_{k}=\sum_{i=0}^{l}\left(\begin{array}{c}
n-2 i \\
l-i
\end{array}\right) S_{2 i}
$$

Recall that

$$
S_{2 i}=\sum_{1 \leq j_{1}<\cdots<j_{2 i} \leq n}\left(e^{\alpha_{1}}+e^{-\alpha_{1}}\right) \ldots\left(e^{\alpha_{j_{2 i}}}+e^{-\alpha_{j_{2 i}}}\right) .
$$

Again, after we choose $\epsilon_{\theta}=1$, we obtain for the product of the twisted character of $K \theta$ and its character on $\sum_{i}(-1)^{i} i \wedge^{i} \mathrm{p}^{*}$ the following expression:

$$
(-1)^{n} 2(2 n+1)\left(\frac{\sum_{i=0}^{l}\left(\begin{array}{c}
n-2 i \\
l-i
\end{array}\right) S_{2 i}}{\prod_{j=1}^{n}\left(e^{\alpha_{j} / 2}-e^{-\alpha_{j} / 2}\right)}\right) \prod_{j=1}^{n}\left(e^{\alpha_{j} / 2}+e^{-\alpha_{j} / 2}\right)^{2} .
$$

We can rewrite this formula as:

$$
\begin{aligned}
& (-1)^{n} 2(2 n+1) \sum_{i=0}^{l}\left(\begin{array}{c}
n-2 i \\
l-i
\end{array}\right) \\
& \times \sum_{1 \leq j_{1}<\cdots<j_{2 i} \leq n}\left(\left(e^{\alpha_{j_{1}}}+e^{-\alpha_{j_{1}}}\right) \ldots\left(e^{\alpha_{j_{2 i}}}+e^{-\alpha_{j_{2 i}}}\right) \prod_{j=1}^{n} \frac{\left(e^{\alpha_{j} / 2}+e^{-\alpha_{j} / 2}\right)^{2}}{\left(e^{\alpha_{j} / 2}-e^{-\alpha_{j} / 2}\right)}\right) .
\end{aligned}
$$

We will denote each set of indices $\left\{j_{1}, \ldots, j_{2 i}\right\}$ appearing in $S_{2 i}$ as $J_{2 i}$. Then the second sum in equation (6.8) can be taken over all possible $J_{2 i}$ 's. For each such $J_{2 i}$ let us regroup the terms of the summand in the following way:

$$
\begin{aligned}
& \left(\prod_{j \in J_{2 i}} \frac{e^{3 / 2 \alpha_{j}}+e^{-3 / 2 \alpha_{j}}+e^{\alpha_{j} / 2}+e^{-\alpha_{j} / 2}}{e^{\alpha_{j} / 2}-e^{-\alpha_{j} / 2}}\left(e^{\alpha_{j} / 2}+e^{-\alpha_{j} / 2}\right)\right) \\
& \times \prod_{j \in J \backslash J_{2 i}} \frac{e^{\alpha_{j} / 2}+e^{-\alpha_{j} / 2}}{e^{\alpha_{j} / 2}-e^{-\alpha_{j} / 2}}\left(e^{\alpha_{j} / 2}+e^{-\alpha_{j} / 2}\right) .
\end{aligned}
$$

Again we identify the first term of each factor as a character formula involving the discrete series representations of $S L(2, \mathbb{R})$. The expression

$$
\frac{e^{3 / 2 \alpha_{j}}+e^{-3 / 2 \alpha_{j}}+e^{\alpha_{j} / 2}+e^{-\alpha_{j} / 2}}{e^{\alpha_{j} / 2}-e^{-\alpha_{j} / 2}}
$$


can be viewed as $\Theta_{\mathcal{D}_{2}^{+}}-\Theta_{\mathcal{D}_{2}^{-}}+\Theta_{\mathcal{D}_{4}^{+}}-\Theta_{\mathcal{D}_{4}^{-}}$with $\Theta$ denoting the characters of the discrete series representations determined by its subscript (see p. 345 in [13]). Just as in the case of the trivial representation, we have that

$$
\frac{e^{\alpha_{j} / 2}+e^{-\alpha_{j} / 2}}{e^{\alpha_{j} / 2}-e^{-\alpha_{j} / 2}}=\Theta_{\mathcal{D}_{2}^{+}}-\Theta_{\mathcal{D}_{2}^{-}}
$$

In both case when we multiply with the factor $e^{\alpha_{j} / 2}+e^{-\alpha_{j} / 2}$ we look at $S O(2)$-types to conclude that the coefficient of the trivial representation is equal to 0 for every $j$. Therefore, when we sum up all the products of zeros, we get that the coefficient of the trivial representation is simply 0 .

Since our choice of $k$ was arbitrary, this argument shows that for every irreducible representation of $M$ in the set $\mathcal{M}_{\sigma}$, we get that the twisted torsion equals 0 . This concludes the proof of the Vanishing Theorem 3.1.1 for the family of groups $S_{0}(2 n+$ $1,1)$.

\section{REFERENCES}

[1] J. Arthur And L. Clozel, Simple Algebras, Base Change, and the Advanced Theory of the Trace Formula, Annals of Mathematics Studies, 120 (1989), Princeton University Press, Princeton, NJ.

[2] A. Borel and N. Wallach, Continuous Cohomology, Discrete Subgroups, and Representations of Reductive Groups, Ann. Math. Studies, 94 (1980), Princeton Univ. Press.

[3] A. Bouaziz, Sur les caractères des groupes de Lie réductifs non connexes, J. Funct. Anal., 70 (1987), pp. 1-79.

[4] T. Bröcker AND T. TOM Dieck, Representations of Compact Lie Groups, Graduate Texts in Mathematics, 98 (1985), Springer-Verlag, New York-Berlin.

[5] J. CheEger, Analytic torsion and the heat equation, Ann. Math., 109 (1972), pp. 259-322.

[6] L. ClOzEL, Changement de base pour les représentations tempérées des groupes réductifs réels, Ann. Scient. École Norm. Sup., (4) 15 (1982), pp. 45-115.

[7] D. FrIED, Analytic torsion and closed geodesics on hyperbolic manifolds, Invent. Math., 84 (1986), no. 3, pp. 523-540.

[8] W. Fulton and J. Harris, Representation Theory: A First Course, Graduate Texts in Mathematics, 129 (1991), Readings in Mathematics, Springer-Verlag, New York.

[9] I. M. Gel'fand, M. I. Graev, I. Piateckil-Shapiro, Representation Theory and Automorphic Functions, W.B. Saunders Co., 1969.

[10] P. Gilkey, Invariance theory, the Heat Equation, and the Atiyah-Singer Index Theorem, Second edition, Studies in Advanced Mathematics, CRC Press, Boca Raton, FL, 1995.

[11] S. Helgason, Differential Geometry, Lie Groups, and Symmetric Spaces, Academic Press, 1978.

[12] A. W. KNAPP, Lie Groups Beyond an Introduction, Progress in Mathematics, Volume 140, Birkhäuser, 1996.

[13] A. W. KNAPP, Representation Theory of Semisimple Groups: An Overview Based on Examples, Princeton Mathematical Series, 36, Princeton University Press, 1986.

[14] A. W. KNAPP AND E.M. STEIN, Intertwining operators for semisimple groups, Ann. of Math., (2) 93 (1971), pp. 489-578.

[15] I. G. Macdonald, Symmetric Functions and Hall Polynomials, Second edition, Oxford Mathematical Monographs, Oxford Science Publications, The Clarendon Press, Oxford University Press, New York, 1995.

[16] H. Moscovici AND R.J. Stanton, Eta-invariants of Dirac operators on locally symmetric manifolds, Inv. Math., 95 (1989), pp. 629-666.

[17] W. MüLLER, Analytic torsion and R-torsion on Riemannian manifolds, Adv. in Math, 28 (1978), pp. 233-305.

[18] D. RAY AND I. Singer, R-torsion and the Laplacian on Riemannian submanifolds, Adv. Math., 7 (1971), pp. 145-210.

[19] J. ROHLFS AND B. SPEH, Representations with cohomology in the discrete spectrum of subgroups of $S O(n, 1)(\mathbb{Z})$ and Lefschetz numbers, Ann. scient. Éc. Norm. Sup., $4^{e}$ série, 20 (1987), pp. $89-136$. 
[20] J. Rohlfs AND B. Speh, A comparison of geometric theta functions for forms of orthogonal groups, Geometry and representation theory of real and p-adic groups, Progress in Mathematics, 158, Birkhäuser-Verlag, 1998.

[21] S. Rosenberg, The Laplacian on a Riemannian Manifold, London Mathematical Society Student Texts 31, Cambridge University Press, New York, 1997.

[22] B. SPEH, Analytic torsion and automorphic forms, Noncompact Lie Groups and their Applications, NATO Adv. Sci. Inst. Ser. C Math. Phys Sci. 429, Kluwer, 1994.

[23] D. A. Vogan, JR., Representations of Real Reductive Lie Groups, Progress in Mathematics, 15, Birkhäuser, Boston, Mass., 1981.

[24] F. WARNER, Introduction to Differentiable Manifolds and Lie Groups, Graduate Texts in Mathematics, 94, Springer-Verlag, New York-Berlin, 1983.

[25] G. Warner, Harmonic Analysis on Semi-simple Lie Groups I. Die Grundlehren der mathematischen Wissenschaften, Band 188. Springer-Verlag, New York-Heidelberg, 1972.

[26] G. WARNER, Harmonic Analysis on Semi-simple Lie Groups II, Die Grundlehren der mathematischen Wissenschaften, Band 189, Springer-Verlag, New York-Heidelberg, 1972. 
M. FUNG 